\title{
New species of semi-aquatic freshwater earthworm genus Glyphidrilus Horst, 1889 from Thailand and Laos (Oligochaeta, Almidae)
}

\author{
Ratmanee Chanabun ${ }^{1,2}$, Khamla Inkavilay ${ }^{3}$, Somsak Panha ${ }^{2}$ \\ I Program in Animal Science, Faculty of Agriculture Technology, Sakon Nakhon Rajabhat University, Sakon \\ Nakhon 47000, Thailand 2 Animal Systematics Research Unit, Department of Biology, Faculty of Science, \\ Chulalongkorn University, Bangkok 10330, Thailand 3 Department of Biology, Faculty of Science, National \\ University of Laos, Vientiane, Laos P.D.R. \\ Corresponding author: Somsak Panha (somsak.pan@chula.ac.th) \\ Academic editor: S. James | Received 17 August 2016 | Accepted 31 March 2017 | Published 3 May 2017 \\ http://zoobank.org/48810F76-97B9-40B2-9A75-DB0F98C5311F \\ Citation: Chanabun R, Inkavilay K, Panha S (2017) New species of semi-aquatic freshwater earthworm genus \\ Glyphidrilus Horst, 1889 from Thailand and Laos (Oligochaeta, Almidae). ZooKeys 672: 1-34. https://doi.org/10.3897/ \\ zookeys.672.10212
}

\begin{abstract}
Seven new species of semi-aquatic freshwater earthworms belonging to the genus Glyphidrilus Horst, 1889 are described from Thailand and Laos, Glyphidrilus nanensis Chanabun \& Panha sp. n., G. satunensis Chanabun \& Panha sp. n., G. chiangraiensis Chanabun \& Panha sp. n., G. namphao Chanabun \& Panha sp. n., G. sekongensis Chanabun \& Panha sp. n., G. namdonensis Chanabun \& Panha sp. n., and G. champasakensis Chanabun \& Panha sp. $\mathbf{n}$. The species are characterized by their external and internal morphological characteristics, as well as body sizes. Other morphological character differences between these seven species were also compared, and an identification key is provided. The relationships of the new species to congeners are discussed.
\end{abstract}

\section{Keywords}

Almidae, earthworms, Glyphidrilus, Laos, southeast Asia, Thailand

Copyright Ratmanee Chanabun et al. This is an open access article distributed under the terms of the Creative Commons Attribution License (CC BY 4.0), which permits unrestricted use, distribution, and reproduction in any medium, provided the original author and source are credited. 


\section{Introduction}

The semi-aquatic freshwater earthworm genus Glyphidrilus Horst, 1889 has been known since the nineteenth century. The unique morphological characters of the expanded epidermis at approximately the clitellum position, called "wings", and the absence of prostate glands, the rounded body with a posterior quadrangular shape and the long slender banana-like cocoons are prominent in Glyphidrilus. The semiaquatic habitat between terrestrial and freshwater ecosystems of rivers, streams, canals, ponds, swamps or even in paddy rice systems are also consistent (Horst 1889, 1893, Jamieson 1968, Michaelsen 1896, 1897, 1900, 1902, 1910, 1918, 1922, Rao 1922, Shen and Yeo 2005, Chanabun et al. 2013). Up to now, the records of Glyphidrilus are only from Africa and Asia; however, most described species are from Asia and especially from Southeast Asia. The worms are now becoming threatened because of the modification, pollution, and destruction of their habitats, for example the contamination by chemical agriculture, and the dam constructions in the upper Mekong River (pers. obs.).

Most previous species were described with some illustrations in a format which was frequently poorly interpreted and insufficient in some species. However, in the recent descriptions and redescriptions of several species are mostly from Thailand, and some from Malaysia, Singapore, and Laos, these deficiencies have been corrected. The color images of both animals and habitats together with anatomical illustration details have made improvements in a new description format. The 19 newly described species reported bring the total number to 40 recognized Glyphidrilus species (Chanabun et al. 2011, Chanabun et al. 2012a, Chanabun et al. 2012b, Chanabun et al. 2013, Chanabun and Panha 2015, Jirapatrasilp et al. 2016).

The behavior of animals has been observed, and it was noted that the worms leave their tail tips exposed near surface of their muddy habitats. While submerging they produce casts as do most earthworms (Chanabun et al. 2013). It was also found that north of 12 degrees latitude in Thailand Glyphidrilus appears as mostly adults in the dry to early rainy seasons (March to July), while in the rainy season they mainly appeared as juveniles, especially in the upper parts of Thailand. However, south of 12 degrees latitude, the adults seem to be present all year round (pers. obs.).

Chanabun et al. (2013) interpreted the phylogeography of Glyphidrilus in light of recent hypotheses regarding ancient river drainage patterns, especially the Mekong River and other main basins, plus various habitat types, using morphological characters and genetic data. Enzyme electrophoresis has proved that the closely related species $G$. mekongensis Panha \& Chanabun, 2012 and G. vangviengensis Panha \& Chanabun, 2011 occurring along the lower Mekong River basin are definitely separate biological species; some possible cryptic species are also suggested in the paper (Jirapatrasilp et al. 2015). The present paper provides additional new species with careful morphological descriptions but no additional genetic data. 


\section{Materials and methods}

The systematic and faunistic surveys of Glyphidrilus were conducted in the lower Mekong River basin both in Thailand and Laos (Fig. 1), and some other river systems in Thailand (Fig. 2) from June 2012 to April 2014. The collections were made by carefully digging up the topsoil near casts on the shore and in the water using hand sorting and sieving the soil from river banks. Adults, juveniles, and cocoons were collected and killed in 30\% (v/v) ethanol, transferred to $5 \%(\mathrm{w} / \mathrm{v})$ formalin for fixation in approximately 12 hours, and then transferred to $75 \%(\mathrm{v} / \mathrm{v})$ ethanol for standard preservation and subsequent morphological studies. Duplicate specimens and/or tissue samples were preserved in 95\% ethanol for further molecular and DNA barcoding analyses.

The type series are deposited in the Chulalongkorn University, Museum of Zoology, Bangkok, Thailand (CUMZ). Additional paratypes will be deposited in the Natural History Museum (NHMUK), London, Raffles Museum of Biodiversity Research, National University of Singapore, Singapore (ZRC) and at the Biozentrum Grindel und Zoologisches Museum, University of Hamburg (ZMH).

The descriptions were made from observations under an Olympus SZX16 stereoscopic light microscope. The following external and internal morphological characters were investigated and recorded: body length and segment number; the positions of clitellum and clitellar wings, genital markings, intestinal origin, gizzard, spermathecae, hearts and seminal vesicles. Illustrations were made for the whole body segments and the external and internal characters. The body width and length were measured in both full adults and juveniles, and are presented as the range (min-max) and mean \pm one standard deviation (SD).

Comparative studies of Glyphidrilus type specimens were made at four natural history museums:

CUMZ Chulalongkorn University, Museum of Zoology.

ZRC Raffles Museum of Biodiversity Research, National University of Singapore, Singapore.

NHMUK The Natural History Museum, London.

ZMH Biozentrum Grindel und Zoologisches Museum, University of Hamburg, Germany.

Anatomical abbreviations are as follows (Chanabun et al. 2013):

$\begin{array}{ll}\text { gm } & \text { genital markings } \\ \text { he } & \text { hearts } \\ \text { np } & \text { nephridia } \\ \text { sc } & \text { spermathecae } \\ \text { sv } & \text { seminal vesicles } \\ \text { wi } & \text { wings }\end{array}$




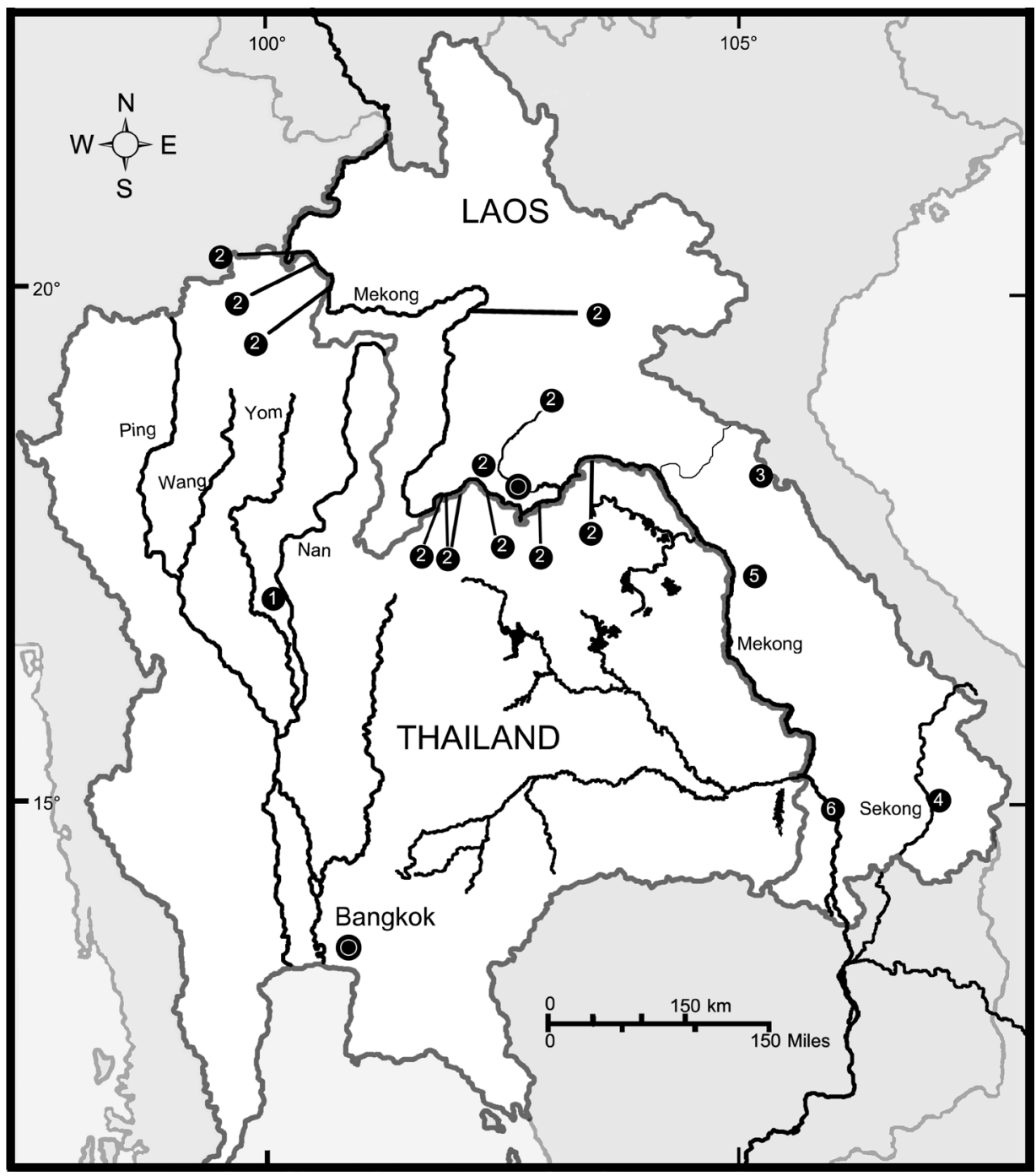

Figure I. Distribution map of the new Glyphidrilus species from Thailand and Laos. Numbers in the circles refer to localities of the new species. I Glyphidrilus nanensis sp. n. 2 Glyphidrilus chiangraiensis sp. n. 3 Glyphidrilus namphao sp. n. 4 Glyphidrilus sekongensis sp. n. 5 Glyphidrilus namdonensis sp. n. and 6 Glyphidrilus champasakensis sp. $\mathrm{n}$.

\section{Systematics}

Family ALMIDAE Duboscq, 1902

\section{Genus Glyphidrilus Horst, 1889}

Diagnosis. Prostomium zygolobous. Body shape nearly circular in cross section in anterior part, and becoming quadrangular in posterior part or after clitellum. Anus 


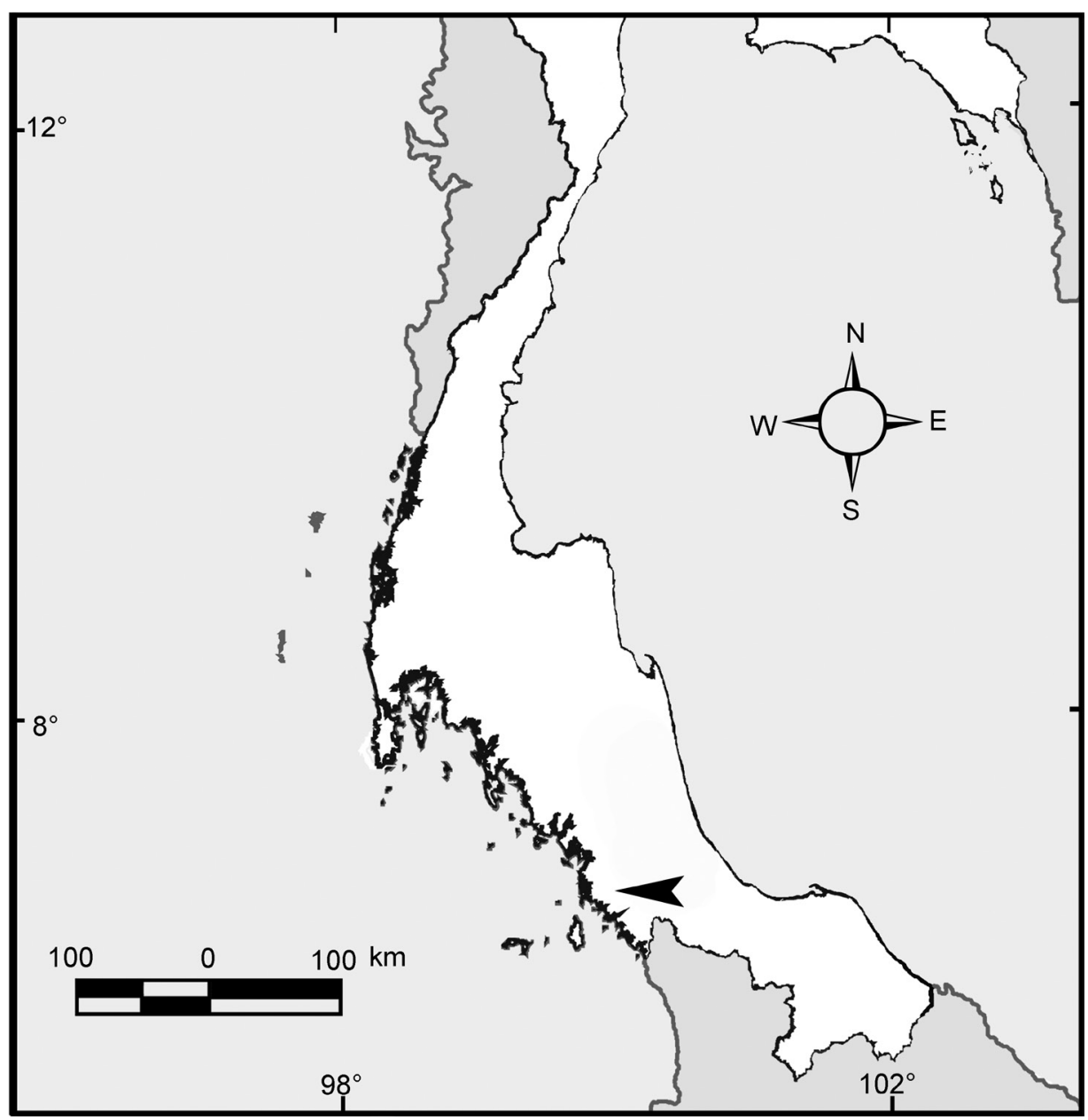

Figure 2. Map showing type locality of Glyphidrilus satunensis sp. n. (arrow head) located on the shore of the Nong Prakpraya at Mueang, Satun, south Thailand.

dorsal or dorso-terminal. A longitudinal lamellar ridge at maturity from body wall on each side in bc, through several of the clitellar segments, which are called wings. Dorsal pores absent. Setae four pairs per segment. Clitellum annular. Genital apertures, all minute and superficial. Male pores inter-or intraclitellar. Spermathecal pores usually all behind the testis segments. Gizzard in VII or VIII sometimes extending into an adjacent segment. Calciferous glands absent. Seminal vesicles usually short, usually four pairs in IX-XII. Holonephridia. Nephrostomes single ducts avesiculate and without sphincters or caeca. Testis and funnels free in X and XI. Male ducts intramural. Ovaries fan shaped and with several egg strings. Ovisacs present or absent. Prostate glands absent and spermathecae without diverticulum (Chanabun et al. 2013, Gates 1972).

Type species. Glyphidrilus weberi Horst, 1889. Type species by original designation in Horst (1889: 77). 


\section{Glyphidrilus nanensis Chanabun \& Panha, sp. n.} http://zoobank.org/E3CF3E43-7045-4C0F-9946-73AAB425425A Figs 1, 3, 4, Table 1

Type material. Holotype: One adult (CUMZ 3403) in a rice field near Nan River at Saklek, Phichit, north Thailand (16 $\left.30^{\prime} 28.4^{\prime \prime} \mathrm{N}, 100^{\circ} 31^{\prime} 15.0^{\prime \prime E}\right), 49$ meters elevation on 16 June 2012. Paratypes: 42 adults and 10 juveniles (CUMZ 3404), 2 adults (ZMH 14579), 2 adults (NHMUK), and 2 adults (ZRC), all same collection data as holotype.

Diagnosis. A small sized earthworm with the clitellar wings on the lateral side of the body in XXIV, XXV, XXVI- XXVII, XXVIII, XXIX. Clitellum in XVII, XVIII-XXXIII, XXXIV. Female pores, male pores, and spermathecal pores not visible. Genital markings: paired or asymmetrical on aa in X, XI, XII, XIII, XIV and XXVIII, XXIX, XXX; paired or asymmetrical on bc in XV, XVI, XVII-XXIII, XXIV, XXV. Four pairs of seminal vesicles in IX-XII. Intestinal origin in XV. Ovaries in XIII-XIV. Spermathecae between 13/14-17/18.

Description of holotype. Dimensions: body length $78 \mathrm{~mm}$, diameter $2.6 \mathrm{~mm}$ in segment VIII, $4.7 \mathrm{~mm}$ before the clitellar wing in segment XXIII, $4.2 \mathrm{~mm}$ after wing in segment XXX in clitellar region; body cylindrical in anterior part, quadrangular in transverse section behind clitellum. 241 segments. Body color pale brown with variations from red to pink at adjacent tissues of wing portion in different individuals of newly collected specimens. At posterior end dorsal surface considerably broader than the ventral. Clitellar wing on ventro-lateral part of clitellum in XXVI-XXIX, $3.6 \mathrm{~mm}$ in height, and $0.3 \mathrm{~mm}$ in width on both sides. Prostomium zygolobous. Dorsal pores absent. Clitellum annular in XVII-XXXIII. Four pairs of setae per segment from II, setal formula aa:ab:bc:cd:dd = 1.0:0.6:1.3:0.6:1.4 in segment VIII and 1.0:0.6:1.5:0.6:1.5 in postclitellar segments. Female pores, male pores, and spermathecal pores not visible. Genital markings: paired on aa in XII-XIII, laterally paired or asymmetrical on bc in XVII-XXIV.

Septa 5/6-8/9 thicker than between other segments, 9/10-14/15 thick and 15/16 to the last segment thin. Gizzard small, globular in 7/8. Intestine enlarged from XV. Hearts, five pairs in VII-XI, first in VII and last in XI. A pair of holonephridia in each segment from segment XIII onwards. Seminal vesicles, four pairs in IX, X, XI, XII. Ovaries, two pairs in XIII-XIV. Testes free in X and XI. Prostate and accessory glands absent. Spermathecae sessile and diverticula absent, elongated oval or globular between $13 / 14-17 / 18$, about $0.2-0.3 \mathrm{~mm}$ in diameter, two to ten on each side per segment.

Variations. Body lengths of adult $(n=49)$ and juvenile paratypes $(n=10)$. Adults ranged from $72-99 \mathrm{~mm}(85.2 \pm 19.0)$, with 186-257 segments. Juveniles ranged from 73-93 mm (82.3 \pm 7.5$)$, with 186-251 segments. Wings in XXIV, XXV, XXVI-XXVII, XXVIII, XXIX, clitellum in XVII, XVIII -XXXIII, XXXIV. Genital markings: paired or asymmetrical on aa in X, XI, XII, XIII, XIV and XXVIII, XXIX, XXX; paired or asymmetrical on bc in XV, XVI, XVII-XXIII, XXIV, XXV.

Distribution. The new species was found in the river banks of Nan River at Saklek, Phichit, north Thailand, which was covered with worm casts. 

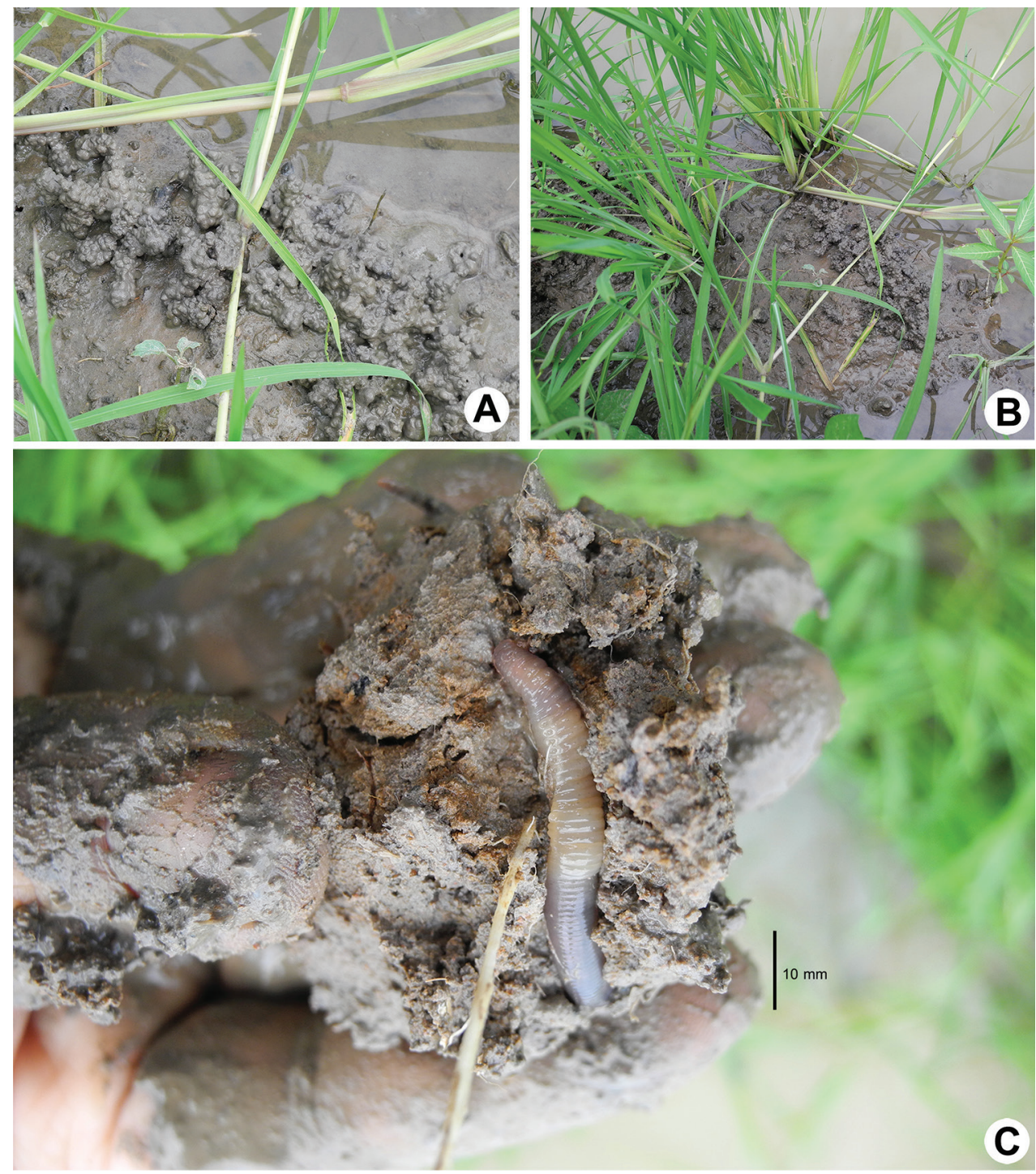

Figure 3. Photographs showing the A Glyphidrilus nanensis sp. n. casts B type locality of G. nanensis sp. n. in the rice field near Nan River at Saklek, Phichit, north Thailand $\mathbf{C}$ coloration of living paratype (CUMZ 3404).

Etymology. The species was named after the locality on the banks of the Nan River. This was the first time that the worm genus was ever recorded from near this river.

Remarks. For a summary of the following comparisons please see Table 1 . The new species differs from G. yunnanensis Chen $\&$ Xu, 1977 reported from China by the latter having longer wings in XXII-XXXII, the clitellum in XVIII-XXXVIII, paired genital markings on bc in XVII-XXI and XXXII-XXXIV, lacking a spermathecae. It differs from G. vangviengensis Chanabun \& Panha, 2011 from Laos by G. vangviengensis 


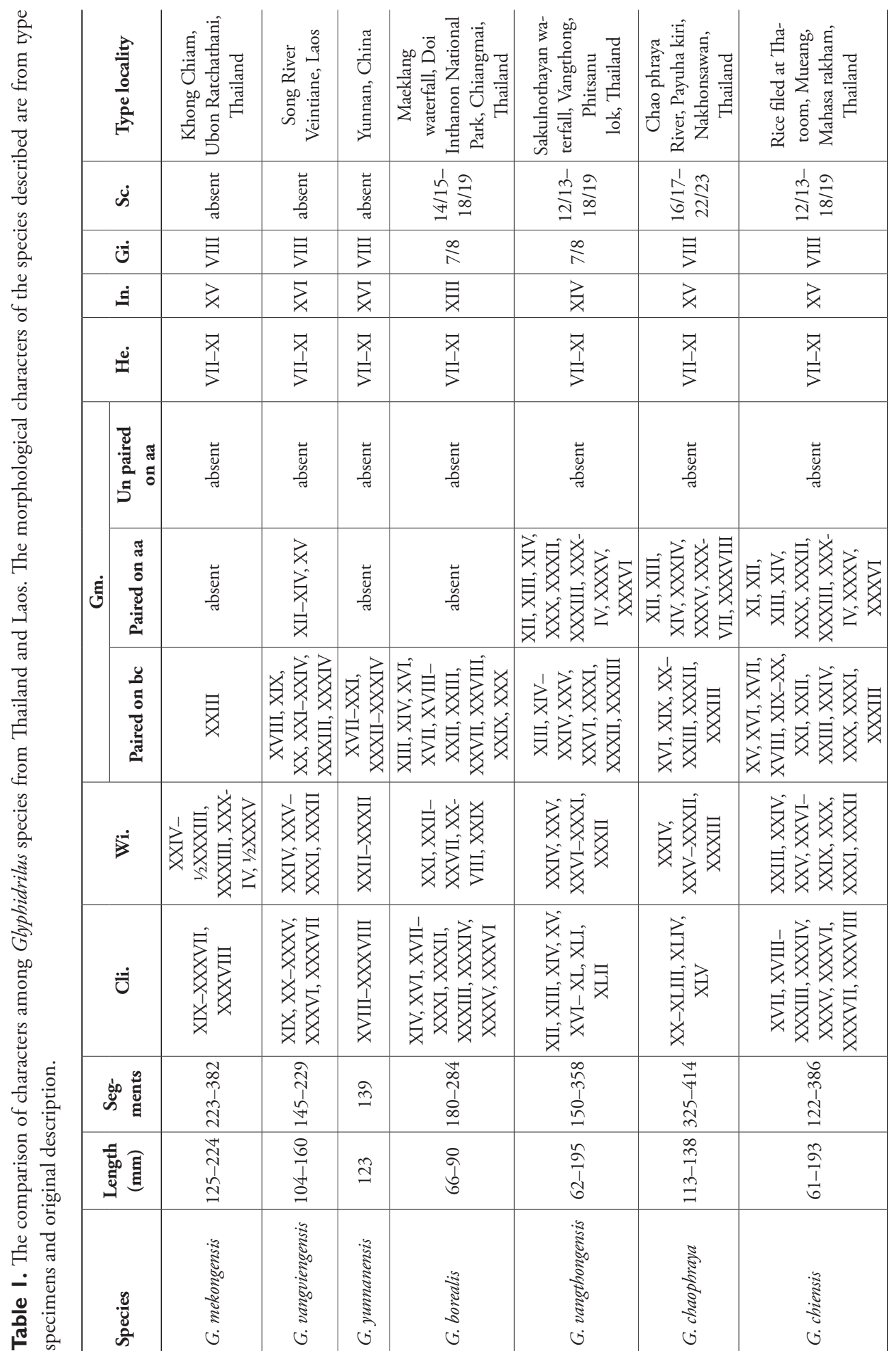




\begin{tabular}{|c|c|c|c|c|c|c|c|}
\hline हू. & 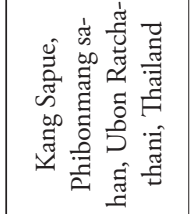 & 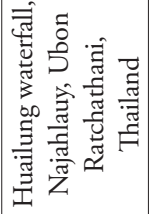 & 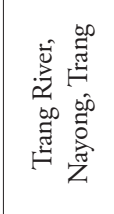 & 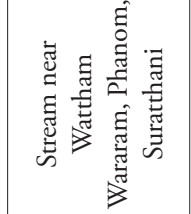 & 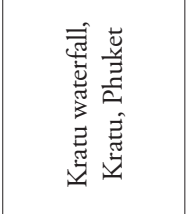 & 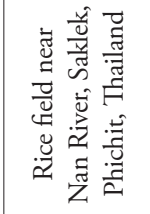 & 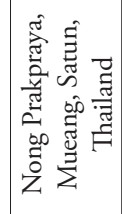 \\
\hline$\dot{\omega}$ & $\stackrel{n}{\stackrel{n}{\beth} \underset{J}{ٍ}}$ & $\begin{array}{l}\text { 苟 } \\
\text { 㟧 }\end{array}$ & 当劣 & $\underset{⿱ 亠}{d} \underset{\sim}{\stackrel{\infty}{\Sigma}}$ & 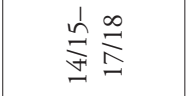 & 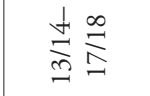 & $\underset{\infty}{\stackrel{d}{a}} \stackrel{0}{\stackrel{n}{n}}$ \\
\hline$\dot{ن}$ & 声 & $\frac{\infty}{n}$ & $\frac{\curvearrowright}{\infty}$ & $\frac{\infty}{\sigma}$ & $\exists$ & $\frac{\infty}{n}$ & 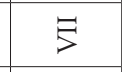 \\
\hline$\doteq$ & $\geqslant$ & 曺 & $\sum$ & 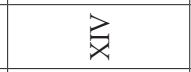 & $\vec{x}$ & $\vec{x}$ & $\sum$ \\
\hline$\stackrel{\text { İ }}{ }$ & 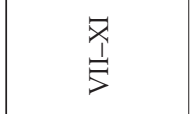 & $\begin{array}{l}\text { 辛 } \\
\text { 泉 }\end{array}$ & 居 & 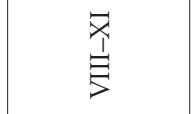 & $\begin{array}{l}\text { 蚱 } \\
\text { 泉 }\end{array}$ & $\begin{array}{l}\underset{X}{1} \\
\stackrel{5}{F}\end{array}$ & $\stackrel{\text { I }}{\stackrel{I}{\prime}}$ \\
\hline 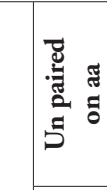 & $\begin{array}{l}\text { 苟 } \\
\text { त् } \\
\text { त्र }\end{array}$ & 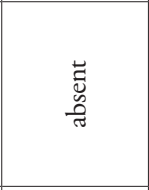 & 紊 & 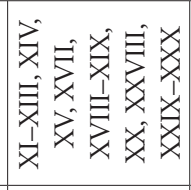 & 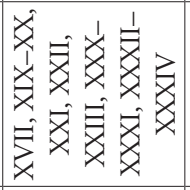 & 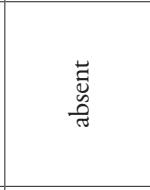 & 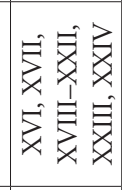 \\
\hline 灾 & 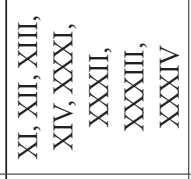 & 番 & $\begin{array}{l}\text { 苟 } \\
\text { तै }\end{array}$ & 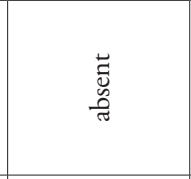 & 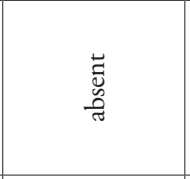 & 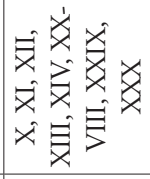 & $\begin{array}{l}\text { च्च } \\
\text { ते } \\
\text { ते }\end{array}$ \\
\hline 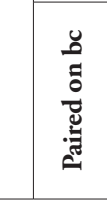 & 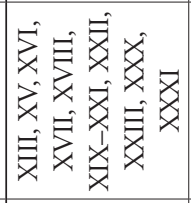 & 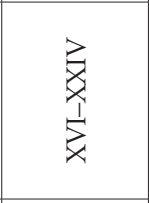 & $\begin{array}{l}\text { 苟 } \\
\text { है }\end{array}$ & 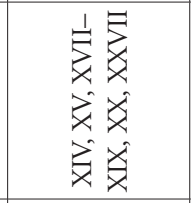 & 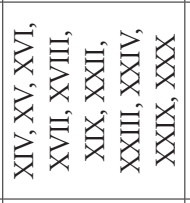 & 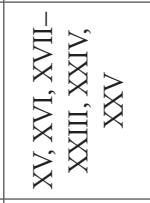 & 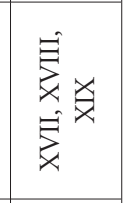 \\
\hline$\dot{\vec{y}}$ & 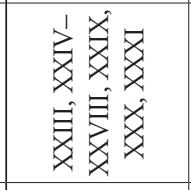 & 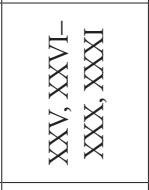 & 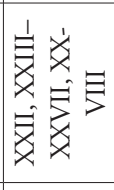 & 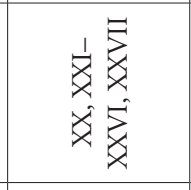 & 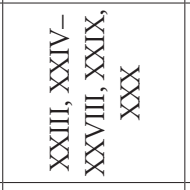 & 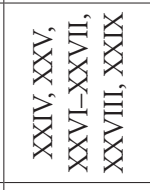 & 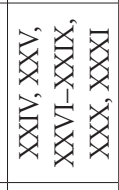 \\
\hline$\ddot{\bar{U}}$ & 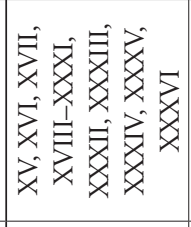 & 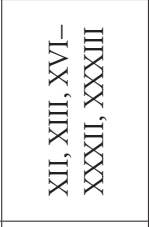 & 齐 & 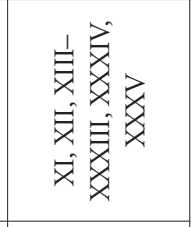 & 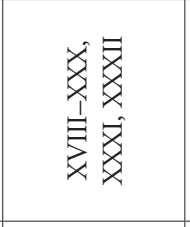 & 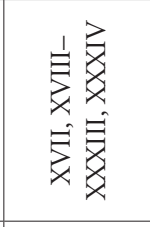 & 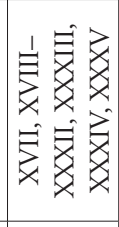 \\
\hline dٌ & $\begin{array}{l}\infty \\
\hat{n} \\
b \\
\infty\end{array}$ & \begin{tabular}{l}
$\infty$ \\
\multirow{1}{1}{} \\
$\stackrel{n}{\sim}$
\end{tabular} & $\begin{array}{c}1 \\
+ \\
+\end{array}$ & 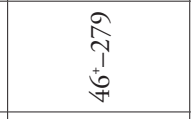 & $\begin{array}{l}\text { No } \\
\stackrel{N}{1} \\
\stackrel{N}{N}\end{array}$ & \begin{tabular}{l}
$\hat{n}$ \\
\multirow{1}{1}{} \\
$\infty$ \\
-
\end{tabular} & $\begin{array}{l}1 \\
\stackrel{1}{\circ} \approx \\
\end{array}$ \\
\hline 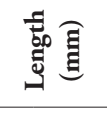 & \begin{tabular}{l}
$\stackrel{0}{n}$ \\
\multirow{n}{n}{} \\
\end{tabular} & $\overrightarrow{\hat{d}}$ & $\begin{array}{l}\stackrel{\ldots}{+} \\
\pm \\
=\end{array}$ & $\begin{array}{l}\stackrel{-}{7} \\
1 \\
\infty \\
\stackrel{\infty}{+} \\
\end{array}$ & 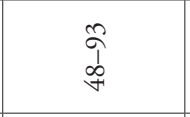 & 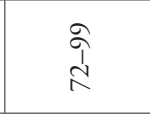 & $\begin{array}{l}\vec{m} \\
\overline{1} \\
0 \\
0\end{array}$ \\
\hline $\begin{array}{l}\text { कूँ } \\
\text { के }\end{array}$ & $\frac{3}{5}$ & 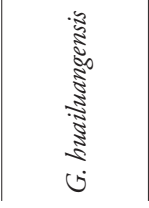 & 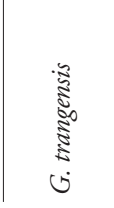 & 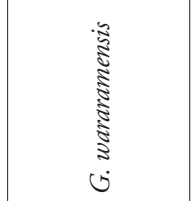 & 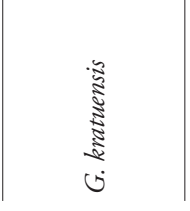 & 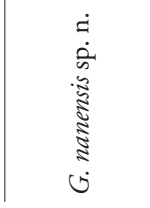 & 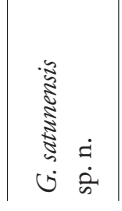 \\
\hline
\end{tabular}




\begin{tabular}{|c|c|c|c|c|c|}
\hline 局 & 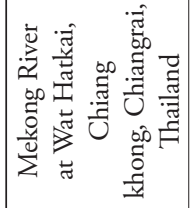 & 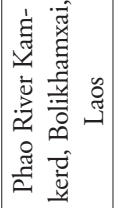 & 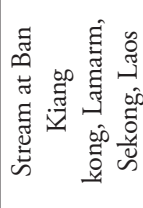 & 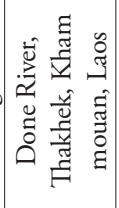 & 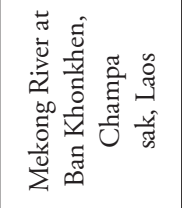 \\
\hline$\dot{~}$ & $\underset{n}{\stackrel{b}{n}} \vec{i}$ & $\underset{\varpi}{ \pm} \underset{\sigma}{Ð}$ & 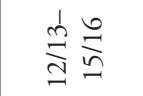 & 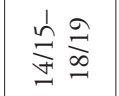 & 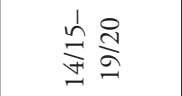 \\
\hline$\dot{ن}$ & 寻 & 寻 & 寻 & 寻 & 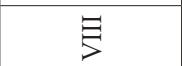 \\
\hline$\dot{\Xi}$ & $\sum$ & $\sum$ & $\sum_{x}$ & $\sum$ & $\sum$ \\
\hline : & 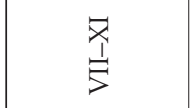 & $\begin{array}{l}\underset{x}{1} \\
\stackrel{F}{F}\end{array}$ & $\stackrel{\text { 尼 }}{\stackrel{1}{\prime}}$ & $\begin{array}{l}\underset{x}{1} \\
\stackrel{5}{>}\end{array}$ & 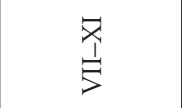 \\
\hline 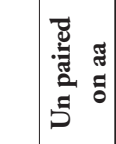 & $\begin{array}{l}\text { 苟 } \\
\text { مै }\end{array}$ & $\begin{array}{l}\text { 苟 } \\
\text { 㫕 }\end{array}$ & 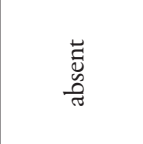 & $\begin{array}{l}\text { 节 } \\
\text { त्र }\end{array}$ & $\begin{array}{l}\text { 苟 } \\
\text { 㫕 }\end{array}$ \\
\hline 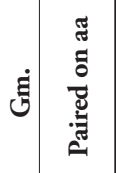 & ${ }_{\substack{x \\
\sum_{x}}}^{\sum_{x}}$ & $\stackrel{\sum}{x}$ & $\begin{array}{l}\text { 䔍 } \\
\text { ले }\end{array}$ & $\begin{array}{l}\vec{x} \\
\dot{\bar{x}}\end{array}$ & 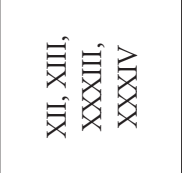 \\
\hline 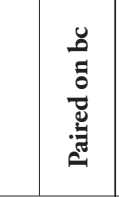 & 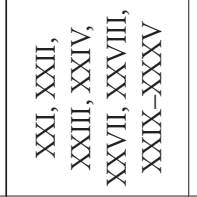 & 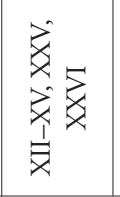 & 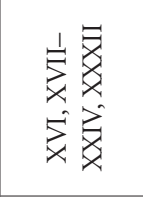 & 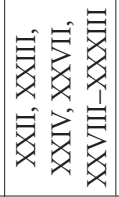 & 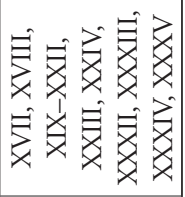 \\
\hline$\dot{\vec{H}}$ & 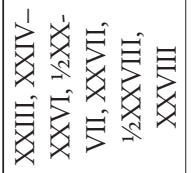 & 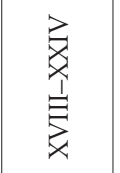 & 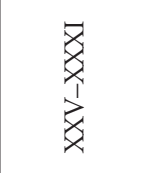 & $\begin{array}{l}1 \\
\text { 紊 } \\
\text { 学 } \\
\text { 紊 }\end{array}$ & 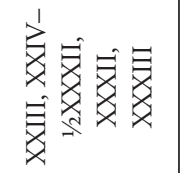 \\
\hline$\dot{\bar{U}}$ & 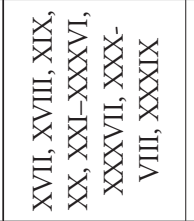 & 㐎㐎 & 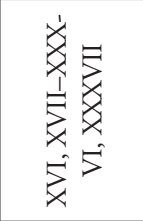 & 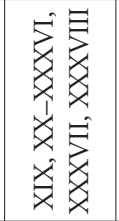 & 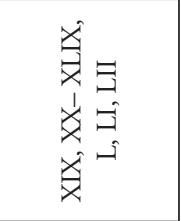 \\
\hline 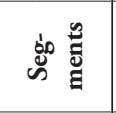 & $\underset{\infty}{\stackrel{1}{1}}$ & 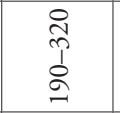 & $\begin{array}{l}\hat{\hat{p}} \\
\hat{\text { ते }}\end{array}$ & 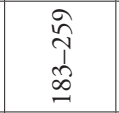 & 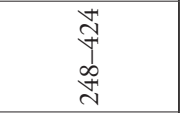 \\
\hline 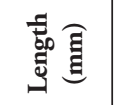 & 离 & \begin{tabular}{l}
\multirow{I}{J}{} \\
\multirow{J}{*}{}
\end{tabular} & $\begin{array}{l}\stackrel{+}{n} \\
\stackrel{2}{a}\end{array}$ & $\frac{a}{a}$ & $\begin{array}{l}\overrightarrow{0} \\
\infty \\
i \\
-0\end{array}$ \\
\hline 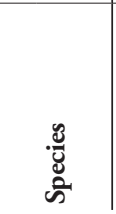 & 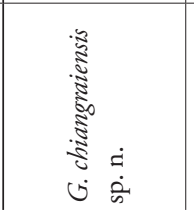 & $\begin{array}{l}\dot{4} \\
\dot{2} \\
8 \\
8 \\
\Sigma \\
\text { है } \\
\vdots \\
\dot{5}\end{array}$ & 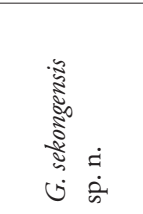 & 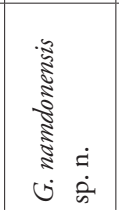 & 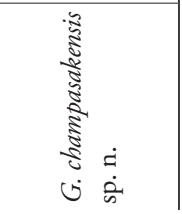 \\
\hline
\end{tabular}




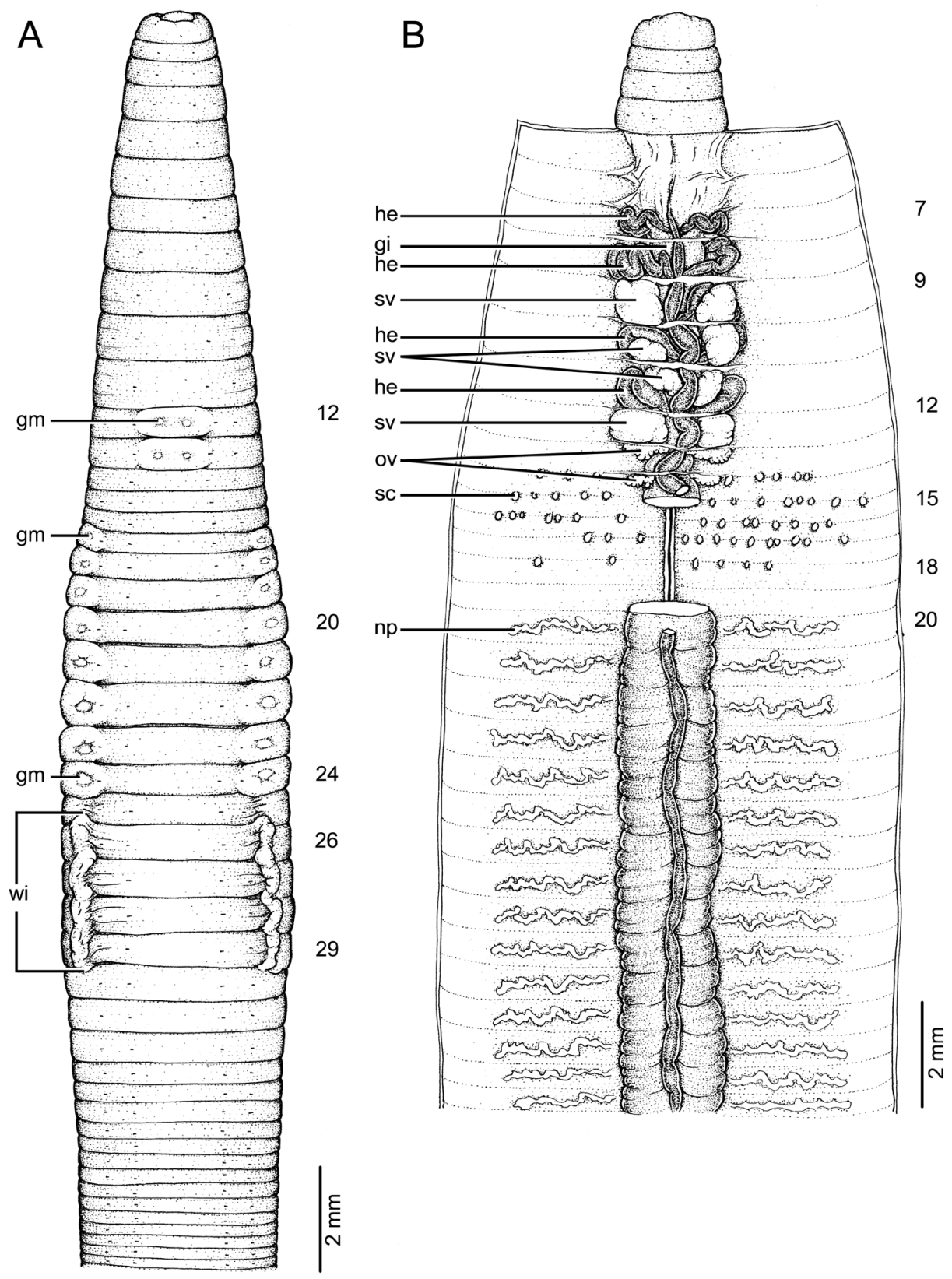

Figure 4. Morphology of holotype (CUMZ 3403) of Glyphidrilus nanensis sp. n. A external ventral view, annular clitellum in XVII-XXXIII B internal dorsal view.

having longer wings in XXIV, XXV-XXXI, XXXII, the clitellum in XIX, XX-XXXV, XXXVI, XXXVII, and lacking spermathecae. It differs from the species recorded as $G$. mekongensis Panha \& Chanabun, 2012 from the Mekong River, Ubon Ratchathani, 
northeast Thailand by the latter species having longer wings in XXIV-1/2XXXIII, XXXIII, XXXIV, 1/2XXXV, the clitellum in XIX-XXXVII, XXXVIII, and lacking spermathecae. It differs from $G$. borealis Chanabun \& Panha, 2013 from Chiangmai, north Thailand by the latter having longer wings in XXI, XXII-XXVII, XXVIII, XXIX, a longer clitellum in XIV, XVI, XVII-XXXI, XXXII, XXXIII, XXXIV, XXXV, XXXVI, and the intestine enlarged from XIII. It differs from $G$. vangthongensis Chanabun \& Panha, 2013 reported from Phitsanulok, north Thailand by G. vangthongensis having longer wings in XXIV, XXV, XXVI-XXXI, XXXII, a longer clitellum in XII, XIII, XIV, XV, XVI-XL, XLI, XLII, an intestine enlarged from XIV, and spermathecae between 12/13-18/19. It differs from $G$. chaophraya Chanabun \& Panha, 2013 from Chaophraya River, Nakhonsawan, central Thailand by the latter having longer wings in XXIV, XXV-XXXII, XXXIII, a longer clitellum in XX-XLIII, XLIV, XLV, and spermathecae between 16/17-22/23. It differs from G. chiensis Chanabun \& Panha, 2013 from Mahasarakham, northeast Thailand by G. chiensis having wings in XXIII, XXIV, XXV, XXVI-XXIX, XXX, XXXI, XXXII, the clitellum in XVII, XVIII-XXXIII, XXXIV, XXXV, XXXVI, XXXVII, XXXVIII, and spermathecae between 12/1318/19. It differs from G. quadratus Chanabun \& Panha, 2013 reported from the Mun River drainage area, northeast Thailand by G. quadratus having wings in XXIII, XXIVXXVIII, XXIX, XXX, XXXI, the clitellum in XV, XVI, XVII, XVIII-XXXI, XXXII, XXXIII, XXXIV, XXXV, XXXVI, and spermathecae between 12/13-17/18. It differs from G. huailuangensis Chanabun \& Panha, 2013 recorded from Najahlauy National Park, Ubon Ratchathani, northeast Thailand which has wings in XXV, XXVI-XXX, XXXI, a clitellum in XII, XIII, XVI-XXXII, XXXIII, and lacking spermathecae. It differs from $G$. sekongensis sp. n. by G. sekongensis sp. n. having longer wings in XXVXXXI, a longer clitellum in XVI, XVII-XXXVI, XXXVII, and spermathecae between 12/13-15/16 (see Table 1).

\section{Glyphidrilus satunensis Chanabun \& Panha, sp. n.}

http://zoobank.org/16A9B4B7-9D5F-409E-B438-059A0FC21BB0

Figs 2, 5, Table 1

Material examined. Holotype: One adult (CUMZ 3405), in Nong Prakpraya, Mueang, Satun, Thailand (06 $\left.44^{\prime} 34.0^{\prime \prime N}, 100^{\circ} 02^{\prime} 23.0^{\prime \prime E}\right), 27$ meters elevation on 16 January 2014. Paratypes: 15 adults and 21 juveniles (CUMZ 3406), 2 adults (ZMH 14580), 2 adults (NHMUK), and 2 adults (ZRC), all same collection data as holotype.

Diagnosis. Glyphidrilus satunensis sp. n. has the clitellar wings on the lateral side of the body in XXIV, XXV, XXVI-XXIX, XXX, XXXI. Clitellum in XVII, XVIIIXXXII, XXXIII, XXXIV, XXXV. Female pores, male pores and spermathecal pores not visible. Genital markings: unpaired on aa in XVI, XVII, XVIII-XXII, XXIII, XXIV; paired or asymmetrical on bc in XVII, XVIII, XIX. Four pairs of seminal vesicles in IX-XII. Intestinal origin in XVI. Ovaries in XIII-XIV. Spermathecae between 13/14-15/16. 

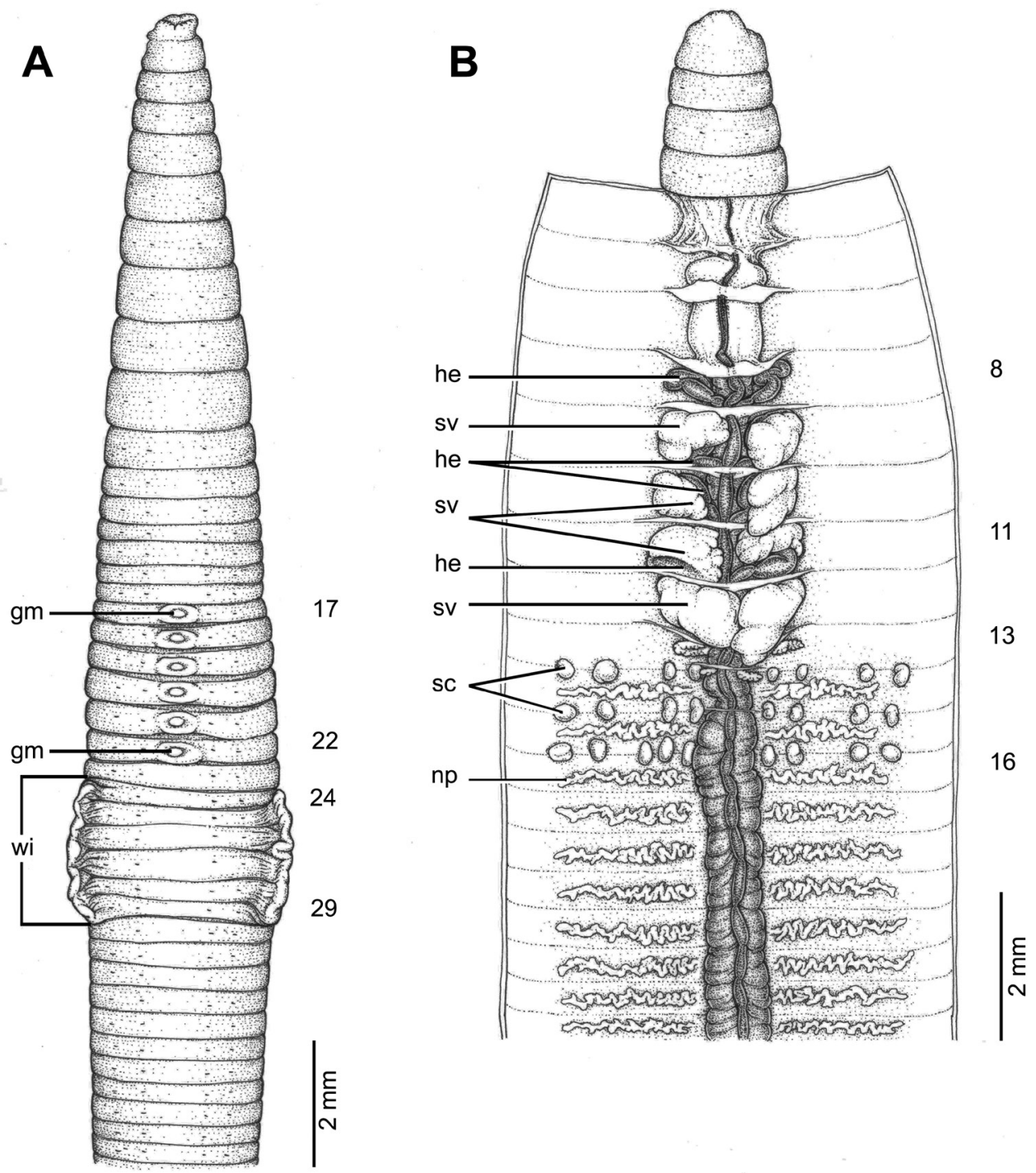

Figure 5. Morphology of holotype (CUMZ 3405) of Glyphidrilus satunensis sp. n. A external ventral view, annular clitellum in XVII-XXXIII B internal dorsal view.

Description of holotype. Dimensions: body length $131 \mathrm{~mm}$, diameter $2.2 \mathrm{~mm}$ in segment VIII, $2.3 \mathrm{~mm}$ before the clitellar wing in segment XXIII, $2.4 \mathrm{~mm}$ after wing in segment XXX in clitellar region; body cylindrical in anterior part, quadrangular in transverse section behind clitellum. 326 segments. Body color pale brown with variations from red to pink at adjacent tissues of wing portion in different individuals of newly collected specimens. At posterior end dorsal surface considerably broader than the ventral. Clitellar wing on ventro-lateral part of clitellum in XXIV-XXIX, $2.5 \mathrm{~mm}$ in height, and $0.5 \mathrm{~mm}$ in width on both sides. Prostomium zygolobous. 
Dorsal pores absent. Clitellum annular in XVII-XXXIII. Four pairs of setae per segment from II, setal formula aa:ab:bc:cd:dd = 1.0:0.5:1.5:1.0:1.5 in segment VIII and 1.0:0.5:1.5:0.5:2.0 in postclitellar segments. Female pores, male pores, and spermathecal pores not visible. Genital markings: unpaired on aa in XVII-XXII.

Septa 5/6-7/8 thicker than between other segments, 8/9-9/10 thick and 10/11 to the last segment thin. Gizzard small, globular in VII. Intestine enlarged from XVI. Hearts, four pairs in VIII-XI. A pair of holonephridia in each segment from segment XIII onwards. Seminal vesicles, four pairs in IX-XII. Ovaries, two pairs in XIII-XIV. Testes free in X and XI. Prostate and accessory glands absent. Spermathecae sessile and diverticula absent, elongated oval or globular between $13 / 14-15 / 16$, about $0.2-0.3$ $\mathrm{mm}$ in diameter, four to five on each side per segment.

Variations. Body lengths of adult $(\mathrm{n}=22)$ and juvenile paratypes $(\mathrm{n}=21)$. Adults ranged from $60^{+}-131 \mathrm{~mm}(95.8 \pm 24.5)$, with $156^{+}-326$ segments. Juveniles ranged from $62-129 \mathrm{~mm}(82.5 \pm 22.1)$, with $166-323$ segments. Wings in XXIV, XXV, XXVI-XXIX, XXX, XXXI, clitellum in XVII, XVIII-XXXII, XXXIII, XXXIV, XXXV. Genital markings: unpaired on aa in XVI, XVII, XVIII-XXII, XXIII, XXIV; paired or asymmetrical on bc in XVII, XVIII, XIX.

Distribution. The new species was found at a pond in Satun, south Thailand.

Etymology. The name "satunensis" is given in reference to the type locality, Satun.

Remarks. See Table 1 for a summary of these comparisons. Glyphidrilus satunensis sp. n. is quite similar to G. kratuensis Chanabun \& Panha, 2013 from Kratu waterfall, Kratu, Phuket, south Thailand in the location of wings but G. kratuensis has a smaller size, a shorter clitellum in XVIII-XXX, XXXI, XXXII, the intestine begins from XIV, and spermathecae are between 14/15-17/18. It differs from G. trangensis Chanabun \& Panha, 2013 from Trang River, Nayong, Trang, south Thailand by the latter having wings in XXII, XXIII-XXVII, XXVIII, the clitellum in XVII, XVIIIXXX, and spermathecae in XVIII-XXI. It differs from G. wararamensis Chanabun \& Panha, 2013 from stream near Wattham Wararam, Phanom, Suratthani, south Thailand by G. wararamensis having wings in XX, XXI-XXVI, XXVII, a clitellum in XI, XII, XIII-XXXIII, XXXIV, XXXV, and spermathecae between 13/14-17/18. It differs from $G$. nanensis sp. n. by $G$. nanensis having a smaller size, slightly shorter wings in XXIV, XXV, XXVI-XXVII, XXVIII, XXIX, unpaired genital markings absent, five pairs of hearts in VII-XI, and spermathecae between 13/14-17/18 (see Table 1).

\section{Glyphidrilus chiangraiensis Chanabun \& Panha, sp. n.} http://zoobank.org/733E54E7-AE95-451F-998F-BC79E71A4155 Figs 1, 6, 7, Table 1

Type material. Holotype: One adult (CUMZ 3407) in the river banks of Mekong River at Wat Hatkai, Chiangkhong, Chiangrai, north Thailand $\left(20^{\circ} 15^{\prime} 8.5^{\prime \prime} \mathrm{N}\right.$, $100^{\circ} 24^{\prime} 46.8^{\prime \prime E}$ ), 384 meters elevation on 14 March 2014. Paratypes: 9 adults (CUMZ 

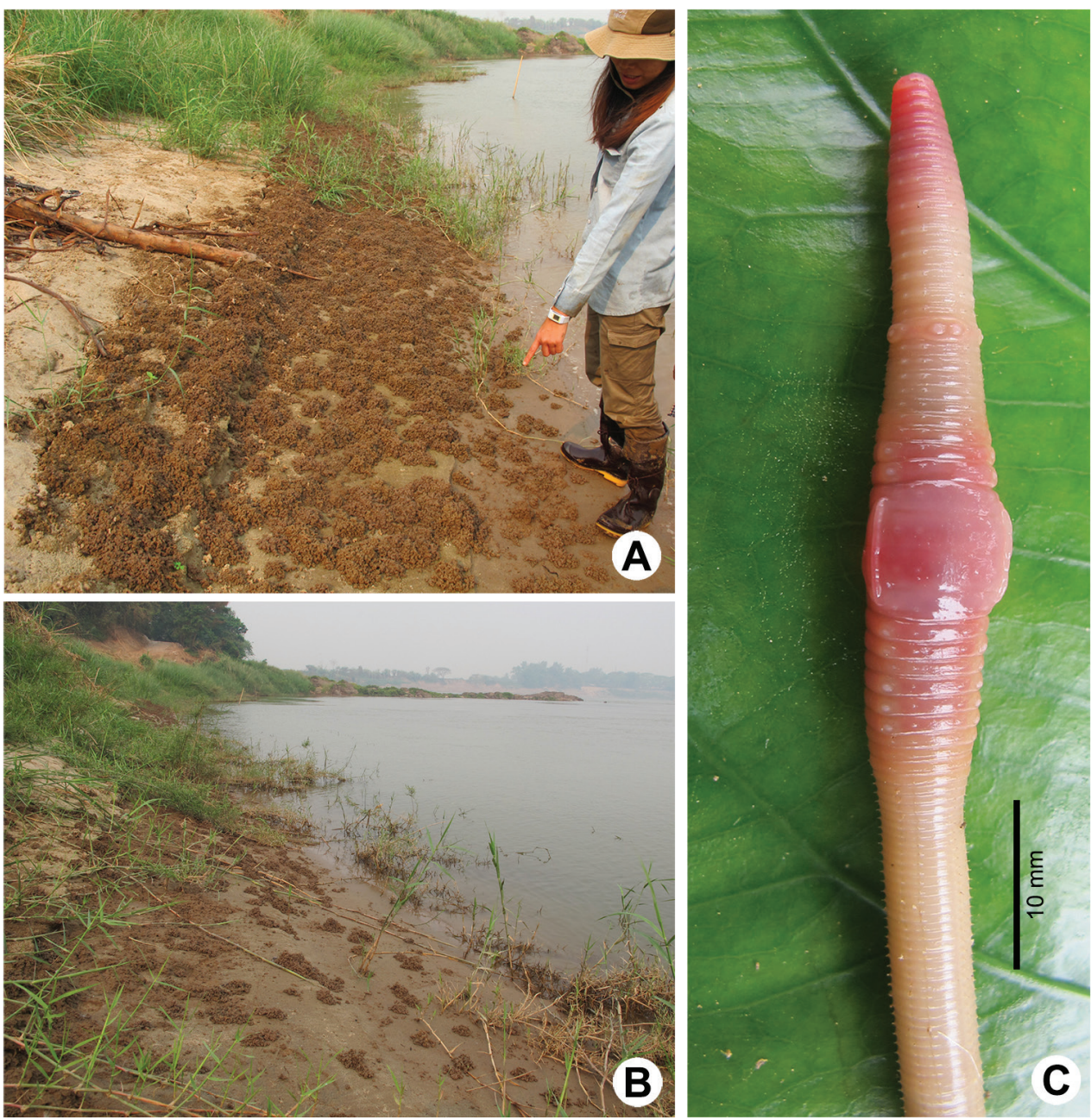

Figure 6. Photographs showing the A Glyphidrilus chiangraiensis sp. n. and other earthworms casts B type locality of $G$. chiangraiensis sp. n. in the river banks of Mekong River at Wat Hatkai, Chiangkhong, Chiangrai, north Thailand, and C coloration of newly collected paratype (CUMZ 3408) after the first preservation step in $30 \%(\mathrm{v} / \mathrm{v})$ ethanol.

3408), 2 adults (ZMH 14581), 2 adults (NHMUK), and 2 adults (ZRC), all specimens collected only from the type locality.

Other material examined. 43 adults (CUMZ 3409), in the river banks of Mekong River at Mueang, Buengkan, northeast Thailand (18²2'2.4"N, 1033' $\left.58.0^{\prime \prime E}\right), 144$ meters elevation on 6 December 2013. 33 adults (CUMZ 3410), in the river banks of Mekong River at Kang Kudku, Chiangkhan, Loei, northeast Thailand (17 $54^{\prime} 24.5^{\prime \prime N}$, $101^{\circ} 42^{\prime} 7.5^{\prime E}$ ), 195 meters elevation on 8 December 2013. 25 adults (CUMZ 3411), in the river banks of Mekong River at Wat Srisomsanook, Chiangkhan, Loei, northeast Thailand $\left(17^{\circ} 59^{\prime} 25.4^{\prime \prime} \mathrm{N}, 101^{\circ} 44^{\prime} 51.3^{\prime \prime E}\right), 212$ meters elevation on 7 December 
2013. 11 adults (CUMZ 3412), in the river banks of Mekong River at Wat Hadphatum, Srichiangmai, Nongkhai, northeast Thailand $\left(17^{\circ} 57^{\prime} 32.2^{\prime \prime} \mathrm{N}, 102^{\circ} 35^{\prime} 26.8^{\prime \prime} \mathrm{E}\right)$, 174 meters elevation on 7 December 2013. 38 adults (CUMZ 3413), in the river banks of Mekong River at Wat Jomnang, Phonphisai, Nongkhai, northeast Thailand (18 01'53.6"N, 1034'47.4"E), 165 meters elevation on 6 December 2013. 30 adults (CUMZ 3414), in the river banks of Mekong River at Wat Prayanakmai, Wiangkaen, Chiangrai, north Thailand $\left(20^{\circ} 11^{\prime} 45.2^{\prime \prime} \mathrm{N}, 100^{\circ} 27^{\prime} 32.0^{\prime \prime} \mathrm{E}\right), 359$ meters elevation on 15 March 2014. 19 adults (CUMZ 3415), in the river banks of Mekong River at Wat Bansaw, Chiangsan, Chiangrai, north Thailand $\left(20^{\circ} 15^{\prime} 19.4^{\prime \prime} \mathrm{N}, 100^{\circ} 10^{\prime} 44.9^{\prime \prime} \mathrm{E}\right)$, 385 meters elevation on 14 March 2014. 26 adults (CUMZ 3416), in river banks of Mekong River at Ban Rimkhong, Pakchom, Loei, northeast Thailand (18 12'48.9"N, $\left.102^{\circ} 04^{\prime} 52.2^{\prime \prime E}\right), 181$ meters elevation on 8 December 2013. 3 adults (CUMZ 3417), in river banks of Mekong River, Mueng Paksay, Chaiyaburi, Laos (18 12'40.0"N, $101^{\circ} 24^{\prime 28.1 " E), ~} 214$ meters elevation on 15 April 2014. 15 adults (CUMZ 3418) in river banks of Mekong River, Bandon, Luangprabang, Laos (1955'27.6"N, $\left.102^{\circ} 10^{\prime} 49.7^{\prime \prime E}\right), 304$ meters elevation on 14 April 2014. 24 adults (CUMZ 3419) in river banks of Mekong River, between Sanakham to Vientiane, Laos (1757'39.7"N, 101²3'53.8"E), 224 meters elevation on 15 April 2014.

Diagnosis. Glyphidrilus chiangraiensis sp. n. has clitellar wings on the lateral side of the body in XXIII, XXIV-XXVI, 1/2XXVII, XXVII, 1/2XXVIII, XXVIII. Clitellum in XVII, XVIII, XIX, XX, XXI-XXXVI, XXXVII, XXXVIII, XXXIX. Female pores, male pores and spermathecal pores not visible. Genital markings: paired or asymmetrical on aa in XII-XIV, XV, XVI, paired or asymmetrical on bc in XXI, XXII, XXIII, XXIV and XXVII, XXVIII, XXIX-XXXV. Four pairs of seminal vesicles in IX-XII. Intestinal origin in XVI. Ovaries in XIII-XIV. Spermathecae between $15 / 16-20 / 21$.

Description of holotype. Dimensions: body length $158 \mathrm{~mm}$, diameter $3.0 \mathrm{~mm}$ in segment VIII, $4.0 \mathrm{~mm}$ before the clitellar wing in segment XXIII, $4.0 \mathrm{~mm}$ after wing in segment XXIX in clitellar region; body cylindrical in anterior part, quadrangular in transverse section behind clitellum. 282 segments. Body color pale brown with variations from red to pink at adjacent tissues of wing portion in different individuals of newly collected specimens. Clitellar wing on ventro-lateral part of clitellum in XXIVXXVII, 1/2XXVIII, $3.7 \mathrm{~mm}$ and $4.0 \mathrm{~mm}$ in height, and $0.5 \mathrm{~mm}$ in width on both sides. Prostomium zygolobous. Dorsal pores absent. Clitellum annular in XVII-XXXVIII. Four pairs of setae per segment from II, setal formula aa:ab:bc:cd:dd=2.0:1.0:2.0:1 0:2.0 in segment VIII and 2.0:1.0:2.0:1.0:2.5 in postclitellar segments. Female pores, male pores, and spermathecal pores not visible. Genital markings: paired on aa in XIV, laterally paired or asymmetrical on bc in XXIII and XXIX-XXXV.

Septa 5/6-8/9 thicker than between other segments, 9/10-13/14 thick and 14/15 to the last segment thin. Gizzard small, globular in VIII. Intestine enlarged from XVI. Hearts, five pairs in VII-XI, first in VII and last in XI. A pair of holonephridia in each segment from segment XIII onwards. Seminal vesicles, four pairs in IX, X, XI, XII. Ovaries, two pairs in XIII and XIV. Testis free in X and XI. Prostate and accessory 

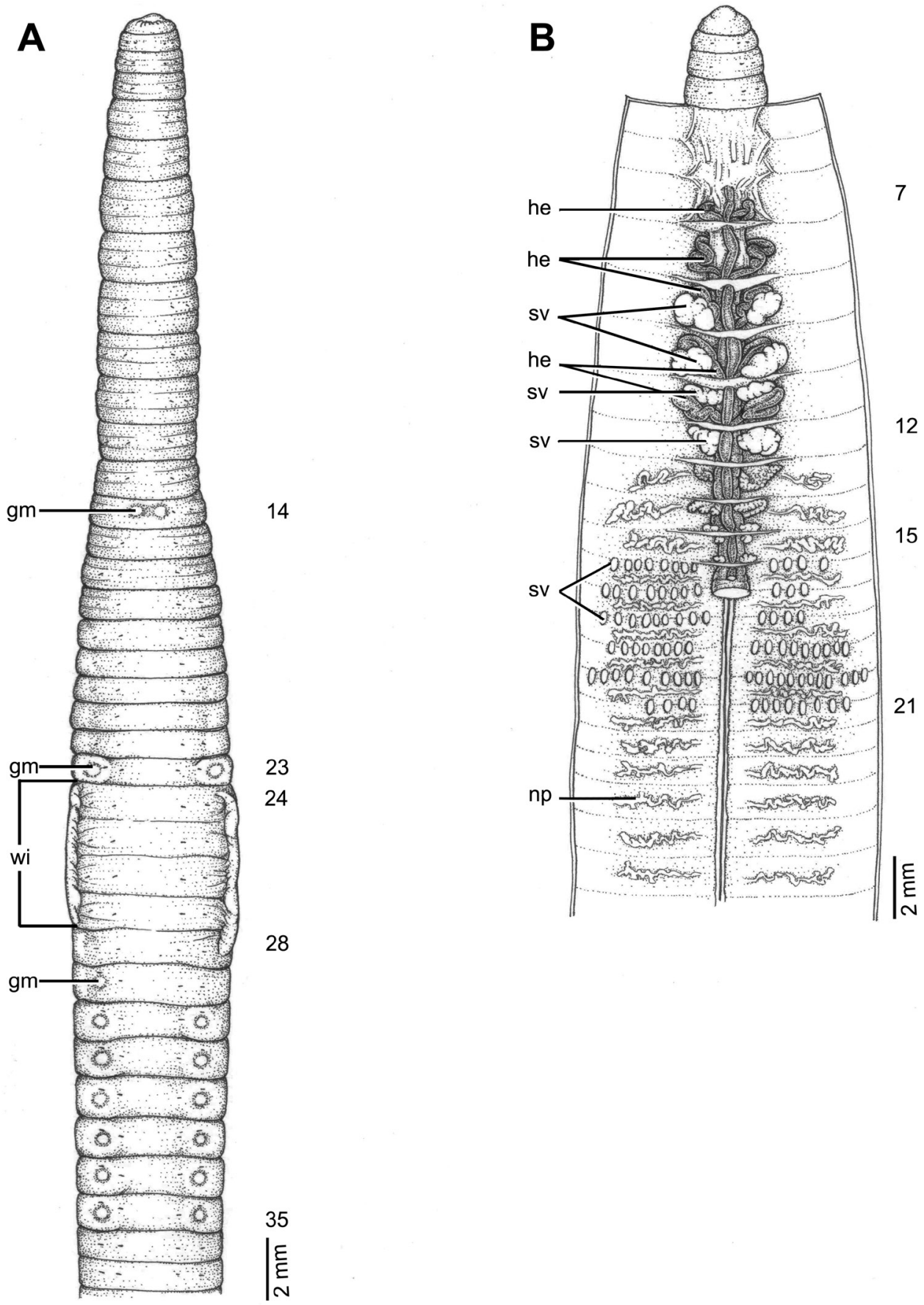

Figure 7. Morphology of holotype (CUMZ 3407) of G. chiangraiensis sp. n. A external ventral view, annular clitellum in XVII-XXXVIII B internal dorsal view. 
glands absent. Spermathecae sessile and diverticula absent, small elongated oval or globular between 15/16-20/21, about $0.1-0.2 \mathrm{~mm}$ in diameter, three to twelve per segment on each side per segment.

Variations. Body lengths of adults $(\mathrm{n}=283)$ ranged from $94-340 \mathrm{~mm}(155.42 \pm$ 54.93), with 89-394 segments. Wings in XXIII, XXXIV-XXVI, 1/2XXVII, XXVII, 1/2XXVIII, XXVIII, clitellum in XVII, XVIII, XIX, XX, XXI-XXXVI, XXXVII, XXXVIII, XXXIX. Genital markings: paired or asymmetrical on aa in XII-XIV, XV, XVI, paired or asymmetrical on bc in XXI, XXII, XXIII, XXIV and XXVII, XXVIII, XXIX-XXXV.

Distribution. The new species is known from the type locality in the river banks of Mekong River at Wat Hatkai, Chiangkhong, Chiangrai, north Thailand, and was found in several locations along the Mekong River and its tributaries in the northeast and north of Thailand at Buengkan, Loei, Nongkhai, and Chiangrai and in Chaiyaburi, Luangprabang, and Sanakham to Vientiane of Laos.

Etymology. The species was named after Chiangrai, the locality name.

Remarks. (see Table 1) Glyphidrilus chiangraiensis sp. n. differs from G. vangviengensis Chanabun \& Panha, 2011 from Song River, Vieintiane, Laos by the latter having longer wings in XXIV, XXV-XXXI, XXXII, the genital markings widely paired in bc XVIII, XIX, XX, XXI-XXIV and XXXIII, XXXIV, paired on aa in XII-XIV, $\mathrm{XV}$ and lacking spermathecae. It differs from G. yunnanensis Chen \& Xu, 1977 reported from China by G. yunnanensis having longer wings in XXII-XXXII, clitellum in XVIII-XXXVIII, and lacking spermathecae. It differs from the species recorded as G. mekongensis Panha \& Chanabun, 2012 from Mekong River, Thailand by $G$. mekongensis having longer wings in XXIV-1/2XXXIII, XXXIII, XXXIV, $1 / 2 X X X V$, and lacking spermathecae. It differs from G. chiensis Chanabun \& Panha, 2013 from Chi River, Mahasarakham, northeast Thailand by G. chiensis having longer wings in XXIII, XXIV, XXV, XXVI-XXIX, XXX, XXXI, XXXII, and spermathecae between 12/13-18/19. It differs from G. quadratus Chanabun \& Panha, 2013 reported from the Mun River by G. quadratus having longer wings in XXIII, XXIV-XXVIII, XXIX, XXX, XXXI, a bit longer clitellum in XV, XVI, XVII, XVIII-XXXI, XXXII, XXXIII, XXXIV, XXXV, XXXVI, and spermathecae between 12/13-17/18. It differs from $G$. huailuangensis Chanabun \& Panha, 2013 recorded from Najahlauy National Park, Ubon Ratchathani, northeast Thailand by the latter having longer wings in XXV, XXVI-XXX, XXXI, clitellum in XII, XIII, XVI-XXXII, XXXIII, and lacks spermathecae. Glyphidrilus chiangraiensis sp. n. differs from G. namphao sp. n. by the latter having wings in XVIII-XXIV, clitellum in XVII-XXVI, XXIX, genital markings: paired on aa in XVII; paired or asymmetrical on bc in XII-XV, XXV, XXVI, and spermathecae between 13/14-16/17. It differs from G. sekongensis sp. n. by the latter having wings in XXV-XXXI, clitellum in XVI, XVII-XXXVI, XXXVII, and spermathecae between 12/13-15/16. It differs from G. champasakensis sp. n. from Mekong River at Ban Khonkhen, Champasak, Laos by the latter having longer wings in XXIII, XXIV-1/2XXXII, XXXII, XXXIII, longer clitellum in XIX, XX-XLIX, L, LI, LII, and spermathecae between 14/15-19/20. 


\section{Glyphidrilus namphao Chanabun \& Panha, sp. n.} http://zoobank.org/D559CDC2-5A05-4D38-93C8-681FB80F3415 Figs 1, 8, Table 1

Type material. Holotype: One adult (CUMZ 3420) in a river banks of Phao River between Ban Lak 7 and Ban Lak 5, Kamkerd, Bolikhamxai, Laos (18 $16^{\prime} 27.7^{\prime N}$, $\left.105^{\circ} 2^{\prime} 44.0 " \mathrm{E}\right), 525$ meters elevation on 7 December 2013. Paratypes: 2 adults and 8 juveniles (CUMZ 3421), 1 adult (ZMH 14582), all specimens collected from only the type locality.

Diagnosis. Glyphidrilus namphao sp. n. is a small worm with the clitellar wings on the lateral side of the body in XVIII-XXIV. Clitellum in XVII-XXVI, XXIX. Female pores, male pores and spermathecal pores not visible. Genital markings: paired or asymmetrical on aa in XVII; paired or asymmetrical on bc in XII-XV and XXV, XXVI. Four pairs of seminal vesicles in IX-XII. Intestinal origin in XVI. Ovaries in XIII-XIV. Spermathecae between 13/14-16/17.

Description of holotype. Dimensions: body length $92 \mathrm{~mm}$, diameter $3.1 \mathrm{~mm}$ in segment VIII, $4.2 \mathrm{~mm}$ before the clitellar wing in segment XVII, $4.2 \mathrm{~mm}$ after wing in segment XXV in clitellar region; body cylindrical in anterior part, quadrangular in transverse section behind clitellum. 269 segments. Body color pale brown with variations from red to pink at adjacent tissues of wing portion in different individuals of newly collected specimens. At posterior end dorsal surface considerably broader than the ventral. Clitellar wing on ventro-lateral part of clitellum in XVIII-XXIV, $3.5 \mathrm{~mm}$ in height, and $0.25 \mathrm{~mm}$ in width on both sides. Prostomium zygolobous. Dorsal pores absent. Clitellum annular in XVII-XXIX. Four pairs of setae per segment from II, setal formula aa:ab:bc:cd:dd $=1.5: 0.5: 1.5: 0.5: 2.0$ in segment VIII and 1.5:0.5:1.5:0.5:2.5 in postclitellar segments. Female pores, male pores, and spermathecal pores not visible. Genital markings: paired on aa in XVII, laterally paired or asymmetrical on bc in XII-XV and XXV.

Septa 4/5-6/7 thicker than between other segments, 7/8-11/12 thick and 12/13 to the last segment thin. Gizzard small, globular in VIII. Intestine enlarged from XVI. Hearts, five pairs in VII-XI, first in VII and last in XI. A pair of holonephridia in each segment from segment XIII onwards. Seminal vesicles, four pairs in IX, X, XI, XII. Ovaries, two pairs in XIII and XIV. Testis free in X and XI. Prostate and accessory glands absent. Spermathecae sessile and diverticula absent, elongated oval or globular between 13/14-16/17, about 0.3-0.4 $\mathrm{mm}$ in diameter, two to seven on each side per segment.

Variations. Body lengths of adult $(n=4)$ and juvenile paratypes $(n=8)$. Adults ranged from 64-122 mm (89.7 \pm 20.8$)$, with 190-320 segments. Juveniles ranged from 64-120 mm (98.3 \pm 17.6$)$, with 193-311 segments. Wings in XVIII-XXIV, clitellum in XVII-XXVI, XXIX. Genital markings: paired or asymmetrical on aa in XVII; paired or asymmetrical on bc in XII-XV and XXV, XXVI.

Distribution. The new species was found in the river banks of Phao River which was covered with worm casts.

Etymology. The species was named after the Phao River, the type locality. 


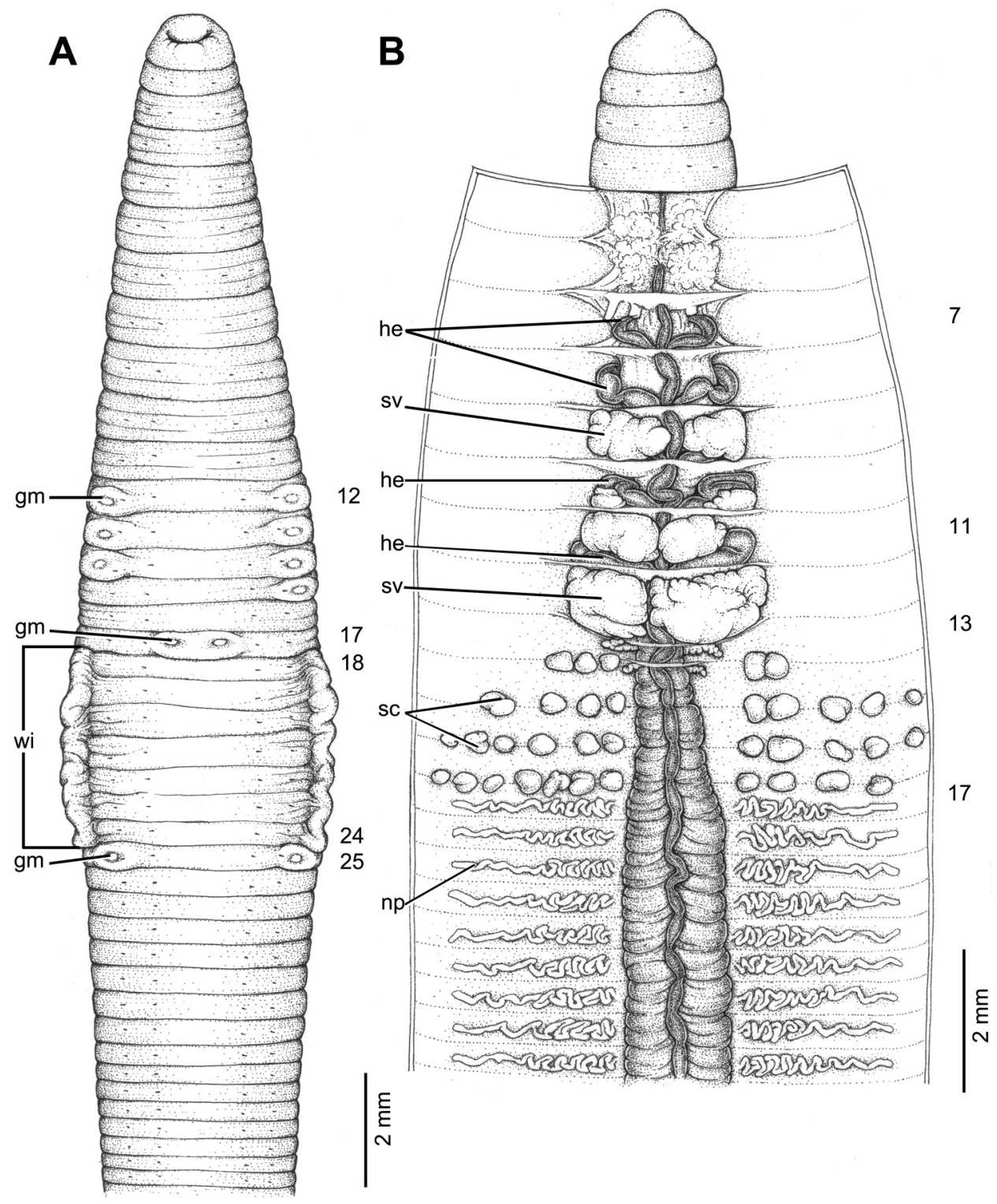

Figure 8. Morphology of holotype (CUMZ 3420) of Glyphidrilus namphao sp. n. A external ventral view, annular clitellum in XVII-XXIX B internal dorsal view.

Remarks. Glyphidrilus namphao sp. n. is different from G. vangviengensis Chanabun \& Panha, 2011 reported from Song River Veintiane, Laos in different locations of wings in XXIV, XXV-XXXI, XXXII, longer clitellum in XIX, XX-XXXV, XXXVI, XXXVII and lacking spermathecae. It differs from $G$. nanensis sp. n. from Nan River at Saklek, Phichit, north Thailand by G. nanensis sp. n. having wings in XXIV, XXV, 
XXVI-XXVII, XXVIII, XXIX, a longer clitellum in XVII, XVIII-XXXIII, XXXIV, and spermathecae between 13/14-17/18. It is different from G. sekongensis sp. n. from Ban Kiangkong, Lamarm, Sekong, Laos in the locations of wings in XXV-XXXI, a longer clitellum in XVI, XVII-XXXVI, XXXVII, the genital markings paired or asymmetrical on bc in XVI, XVII-XXIV, XXXII, and spermathecae between 12/13-15/16. Glyphidrilus namphao sp. n. differs from G. chiangraiensis sp. n. from Mekong River at Wat Hatkai, Chiangkhong, Chiangrai, north Thailand in the locations of wings in XXIII, XXIV-XXVI, 1/2XXVII, XXVII, 1/2XXVIII, XXVIII, a longer clitellum in XVII, XVIII, XIX, XX, XXI-XXXVI, XXXVII, XXXVIII, XXXIX, and spermathecae between 15/16-20/21 (see Table 1).

\section{Glyphidrilus sekongensis Chanabun \& Panha, sp. n.}

http://zoobank.org/F9F57BA6-C6DC-46EB-8D14-9427E9ADD3E7

Figs 1, 9, Table 1

Type material. Holotype: One adult (CUMZ 3422) in a stream at Ban Kiangkong, Lamarm, Sekong, Laos (15³3'30.6"N, 106¹9'19.4"E), 472 meters elevation on 17 October 2013. Paratypes: 2 adults and 11 juveniles (CUMZ 3423) all same collection data as holotype.

Diagnosis. Glyphidrilus sekongensis sp. n. is a small sized earthworm with the clitellar wings on the lateral side of the body in XXV-XXXI. Clitellum in XVI, XVIIXXXVI, XXXVII. Female pores, male pores and spermathecal pores not visible. Genital markings: paired or asymmetrical on bc in XVI, XVII-XXIV and XXXII. Four pairs of seminal vesicles in IX-XII. Intestinal origin in XVI. Ovaries in XIII-XIV. Spermathecae between 12/13-15/16.

Description of holotype. Dimensions: body length $134 \mathrm{~mm}$, diameter $2.4 \mathrm{~mm}$ in segment VIII, $2.3 \mathrm{~mm}$ before the clitellar wing in segment XXIV, $2.4 \mathrm{~mm}$ after wing in segment XXXII in clitellar region; body cylindrical in anterior part, quadrangular in transverse section behind clitellum. 329 segments. Body color pale brown with variations from red to pink on adjacent tissues of wing portions in different individuals of newly collected specimens. The posterior end on the dorsal surface is considerably broader than the ventral. Clitellar wing on ventro-lateral part of clitellum in XXVXXXI, $4.0 \mathrm{~mm}$ in height, and $0.5 \mathrm{~mm}$ in width on both sides. Prostomium zygolobous. Dorsal pores absent. Clitellum annular in XVI-XXXVII. Four pairs of setae per segment from II, setal formula aa:ab:bc:cd:dd = 1.0:0.3:1.0:0.5:1.3 in segment VIII and 1.8:0.8:1.3:0.6:1.5 in postclitellar segments. Female pores, male pores, and spermathecal pores not visible. Genital markings: laterally paired or asymmetrical on bc in XVI-XXIV, XXXII.

Septa 5/6-8/9 thicker than between other segments, 9/10-14/15 thick and 15/16 to the last others segment, $9 / 10-14 / 15$ thick and 15/16 to the last segment thin. Gizzard small, globular in VIII. Intestine enlarged from XVI. Hearts, four pairs in VIIIXI, first in VIII and last in XI. A pair of holonephridia in each segment from segment 

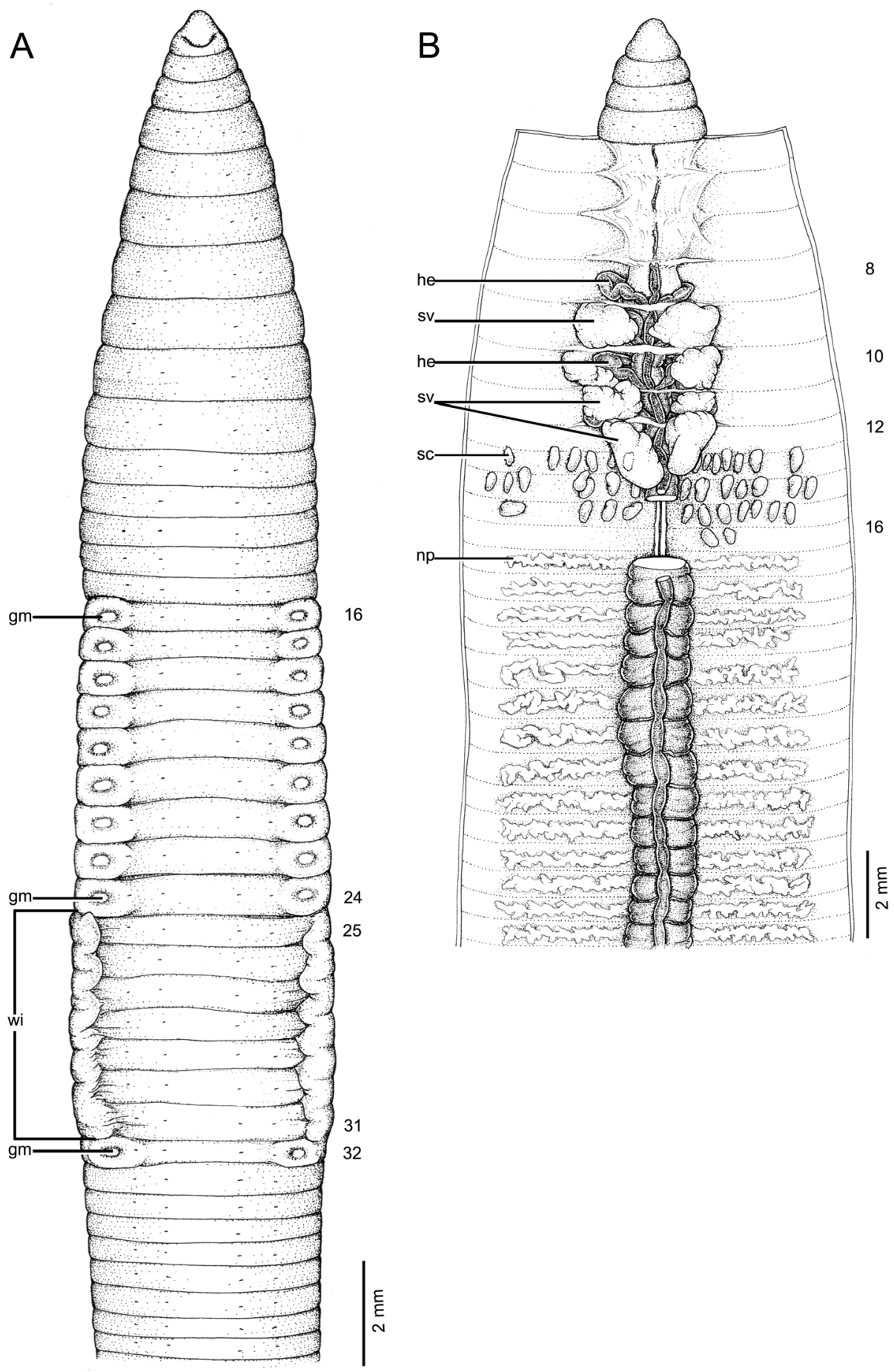

Figure 9. Morphology of holotype (CUMZ 3422) of Glyphidrilus sekongensis sp. n. A external ventral view, annular clitellum in XVI-XXXVII B internal dorsal view. 
XIII onwards. Seminal vesicles, four pairs in IX, X, XI, XII. Ovaries, two pairs in XIII and XIV. Testis free in X and XI. Prostate and accessory glands absent. Spermathecae sessile and diverticula absent, small elongated oval or globular between 12/13-15/16, about $0.1-0.3 \mathrm{~mm}$ in diameter, two to seven on each side per segment.

Variations. Body lengths of adult $(n=3)$ and juvenile paratypes $(n=11)$. Adults ranged from 90-134 mm (109.6 \pm 12.2$)$, with 237-337 segments. Juveniles ranged from $86^{+}-130 \mathrm{~mm}(104.2 \pm 14.0)$, with $209^{+}-329$ segments. Wings in XXV-XXXI, clitellum in XVI, XVII-XXXVI, XXXVII. Genital markings: laterally paired or asymmetrical on bc in XVI, XVII-XXIV, XXXII.

Distribution. The new species was found in a stream at Ban Kiangkong, Lamarm, Sekong, Laos. Soils are slightly sandy mixed with black organic matter.

Etymology. The species was named after Sekong, the type locality in Laos.

Remarks. (see Table 1). Glyphidrilus sekongensis sp. n. differs from other species by the locations of external and internal characteristics such as wings, clitellum, the arrangement of genital markings, and spermathecae. The new species differs from $G$. vangviengensis Chanabun \& Panha, 2011 from Song River, Vientiane, Laos by the latter having a shorter clitellum in XIX, XX-XXXV, XXXVI, XXXVII, widely paired genital markings in bc XVIII, XIX, XX, XXI-XXIV, XXXIII, XXXIV, paired on aa in XII, XIII, XIV, XV, and lacks spermathecae. Differences from $G$. yunnanensis Chen $\& \mathrm{Xu}, 1977$ reported from China are that G. yunnanensis has longer wings in XXIIXXXII, clitellum in XVIII-XXXVIII, and lacks spermathecae. Differences from $G$. mekongensis Panha \& Chanabun, 2012 are that $G$. mekongensis has longer wings in XXIV-1/2XXXIII, XXXIII, XXXIV, 1/2XXXV, and lacks spermathecae. It differs from G. huailuangensis Chanabun \& Panha, 2013 recorded from Najahlauy National Park, Ubon Ratchathani, northeast Thailand by the latter having a larger body size, clitellum in XII, XIII, XVI-XXXII, XXXIII, and lacks spermathecae. It differs from G. champasakensis sp. $\mathrm{n}$. from Mekong River at Ban Khonkhen, Champasak, Laos by the latter having longer wings in XXIII, XXIV_1/2XXXII, XXXII, XXXIII, longer clitellum in XIX, XX-XLIX, L, LI, LII, and spermathecae between 14/15-19/20.

\section{Glyphidrilus namdonensis Chanabun \& Panha, sp. n.} http://zoobank.org/64F05048-0871-4BF0-A7F5-B9FB02701348 Figs 1, 10, Table 1

Type material. Holotype: One adult (CUMZ 3424) in the banks of Done River at Ban Namdone, Thakhek, Khammouan, Laos (17²8'39.9"N, 104²5'35.1"E), 161 meters elevation on 6 December 2013. Paratypes: 16 adults and 6 juveniles (CUMZ 3425), 2 adults (ZMH 14583), 2 adults (NHMUK), and 2 adults (RMBR), all same collection data as holotype.

Diagnosis. Glyphidrilus namdonensis sp. n. is small sized earthworm with distinctly expanded tissues, the clitellar wings on the lateral side of the body in XXIV, XXVXXVIII, XXX. Clitellum in XIX, XX-XXXVI, XXXVII, XXXVIII. Female pores, 

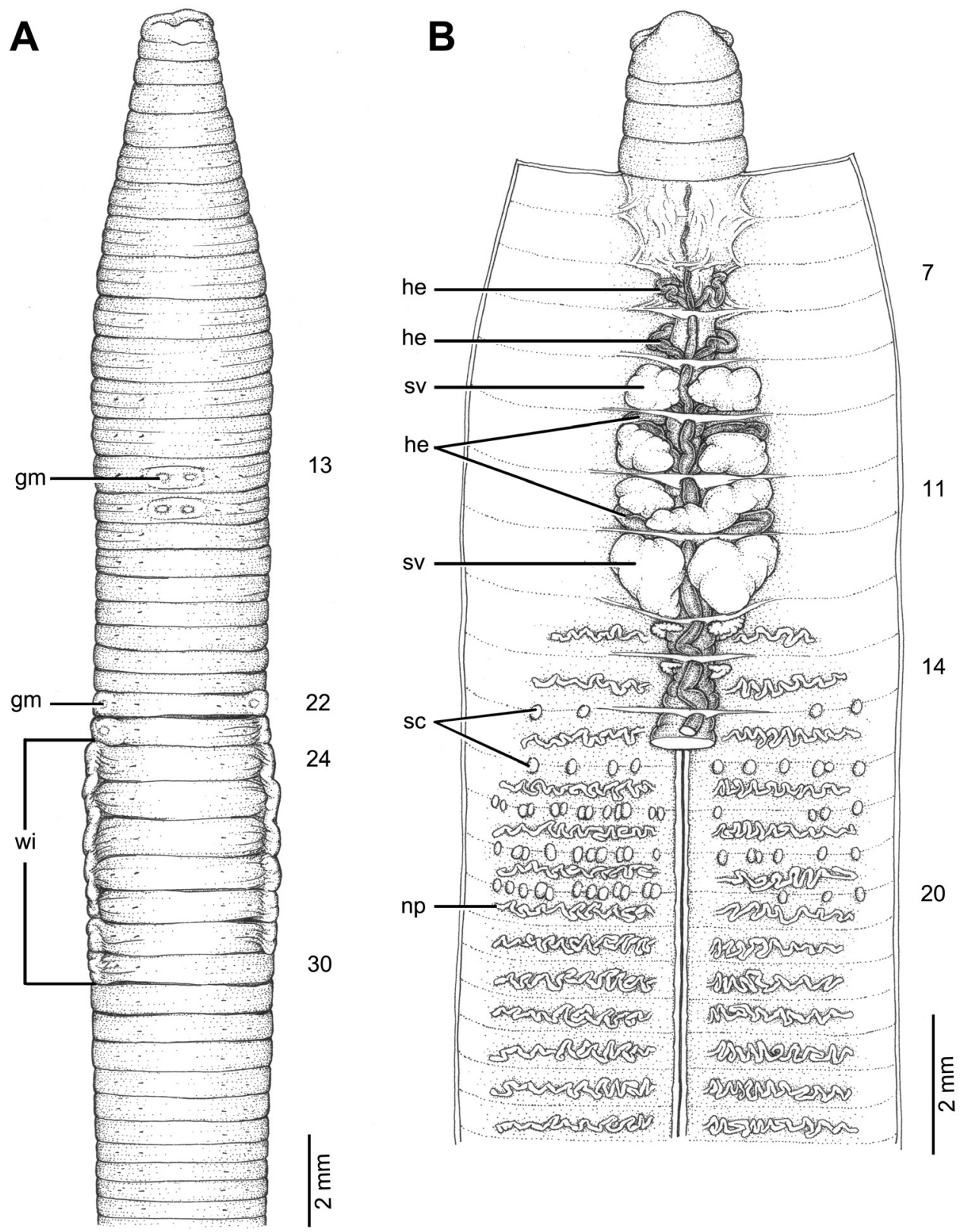

Figure 10. Morphology of holotype (CUMZ 3424) of Glyphidrilus namdonensis sp. n. A external ventral view, annular clitellum in XX-XXXVII B internal dorsal view.

male pores and spermathecal pores not visible. Genital markings: medium paired or asymmetrical on aa in XIII, XIV; paired or asymmetrical on bc in XXII, XXIII, XXIV and XXVII, XXVIII-XXXIII. Four pairs of seminal vesicles in IX-XII. Intestinal origin in XVI. Ovaries in XIII-XIV. Spermathecae between 14/15-18/19. 
Description of holotype. Dimensions: body length $92 \mathrm{~mm}$, diameter $3.5 \mathrm{~mm}$ in segment VIII, $4.0 \mathrm{~mm}$ before the clitellar wing in segment XXIII, $4.0 \mathrm{~mm}$ after the wing in segment XXXI in the clitellar region; body cylindrical in the anterior part, quadrangular in transverse section behind clitellum. 216 segments. Body color pale brown with variations from red to pink in adjacent tissues of the wing portions in different individuals of newly collected specimens. Clitellar wing on ventro-lateral part of clitellum in XXIV-XXX, $5.0 \mathrm{~mm}$ in height, and $0.2 \mathrm{~mm}$ in width on both sides. Prostomium zygolobous. Dorsal pores absent. Clitellum annular in XX-XXXVII. Four pairs of setae per segment from II, setal formula aa:ab:bc:cd:dd = 1.3:0.5:1.0:0.5:2.0 in segment VIII and 1.5:0.5:1.0:0.5:2.3 in postclitellar segments. Female pores, male pores, and spermathecal pores not visible. Genital markings: medium paired on aa in XIII, XIV, laterally paired or asymmetrical on bc in XXII, XXIII.

Septa 5/6-8/9 thicker than between other segments, 9/10-14/15 thick, and 15/16 to the last segment thin. Gizzard small, globular in VIII. Intestine enlarged from XVI. Hearts, five pairs in VII-XI, first in VII and last in XI. A pair of holonephridia in each segment from segment XII onwards. Seminal vesicles, four pairs in IX, X, XI, XII. Ovaries two pairs in XIII and XIV. Testis free in X and XI. Prostate and accessory glands absent. Spermathecae sessile and diverticula absent, small elongated oval or globular between $14 / 15-18 / 19$, about $0.1-0.2 \mathrm{~mm}$ in diameter, two to thirteen on each side per segment.

Variations. Body lengths of adult $(n=23)$ and juvenile paratypes $(n=6)$. Adults ranged from $90-139 \mathrm{~mm}(121.9 \pm 25.9)$, with $183-259$ segments. Juveniles ranged from 87-136 mm (103.3 \pm 17.4$)$, with 185-223 segments. Wings in XXIV, XXVXXVIII, XXX, clitellum in XIX, XX-XXXVI, XXXVII, XXXVIII. Genital markings: medium paired or asymmetrical on aa in XIII, XIV; paired or asymmetrical on bc in XXII, XXIII, XXIV and XXVII, XXVIII-XXXIII.

Distribution. The new species was found only from the banks of Done River at Ban Namdone, Thakhek, Khammouan, Laos.

Etymology. The species was named after the Done River.

Remarks. Glyphidrilus namdonensis sp. n. is compared with other Glyphidrilus having spermathecae and a clitellum starting feom segment XIX. Glyphidrilus namdonensis differs from G. champasakensis sp. $\mathrm{n}$. by the latter having longer wings in XXIII, XXIV-1/2XXXII, XXXII, XXXIII, longer clitellum in XIX, XX-XLIX, L, LI, LII, and spermathecae between 14/15-19/20 (see Table 1).

\section{Glyphidrilus champasakensis Chanabun \& Panha, sp. n.} http://zoobank.org/32CACB01-68EE-4093-A66E-8EEF939A3B6D Figs $1,11,12$, Table 1

Type material. Holotype: One adult (CUMZ 3426) in the banks of Mekong River at Ban Khonkhen, Champasak, Laos (15'02'21.2"N, 10551'20.4"E), 106 meters elevation on 17 April 2014. Paratypes: 17 adults (CUMZ 3427), 2 adults (ZMH 14584), 2 adults (NHMUK), and 2 adults (RMBR), all specimens collected from type locality. 

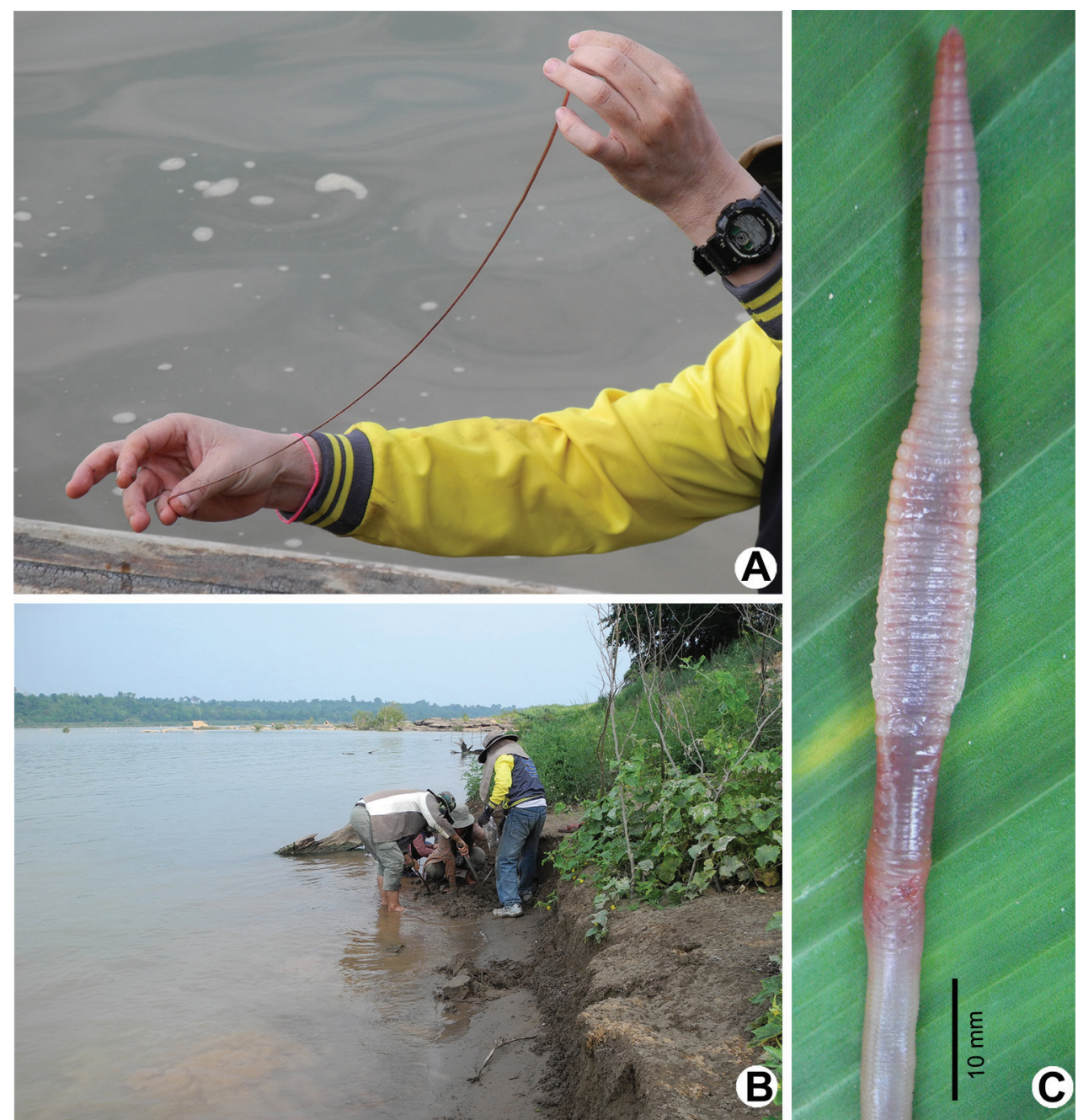

Figure I I. Photographs showing the A Glyphidrilus champasakensis sp. n. B type locality of G. cham-
pasakensis sp. $\mathrm{n}$. in the banks of Mekong River at Ban Khonkhen, Champasak, Laos and $\mathbf{C}$ coloration of newly collected paratype (CUMZ 3427) after the first preservation step in 30\% (v/v) ethanol.

Diagnosis. Glyphidrilus champasakensis sp. n. is a large sized earthworm with distinct expanded tissues of the clitellar wings on the sides of the body in XXIII, XXIV1/2XXXII, XXXII, XXXIII. Clitellum in XIX, XX- XLIX, L, LI, LII. Female pores, male pores and spermathecal pores not visible. Genital markings: medium paired or asymmetrical on aa in XII, XIII, XXXIII, XXXIV; paired or asymmetrical on bc in XVII, XVIII, XIX-XXII, XXIII, XXIV and XXXII, XXXIII, XXXIV, XXXV. Four pairs of seminal vesicles in IX-XII. Intestinal origin in XVI. Large ovaries in XIII-XIV. Spermathecae between 14/15-19/20. 


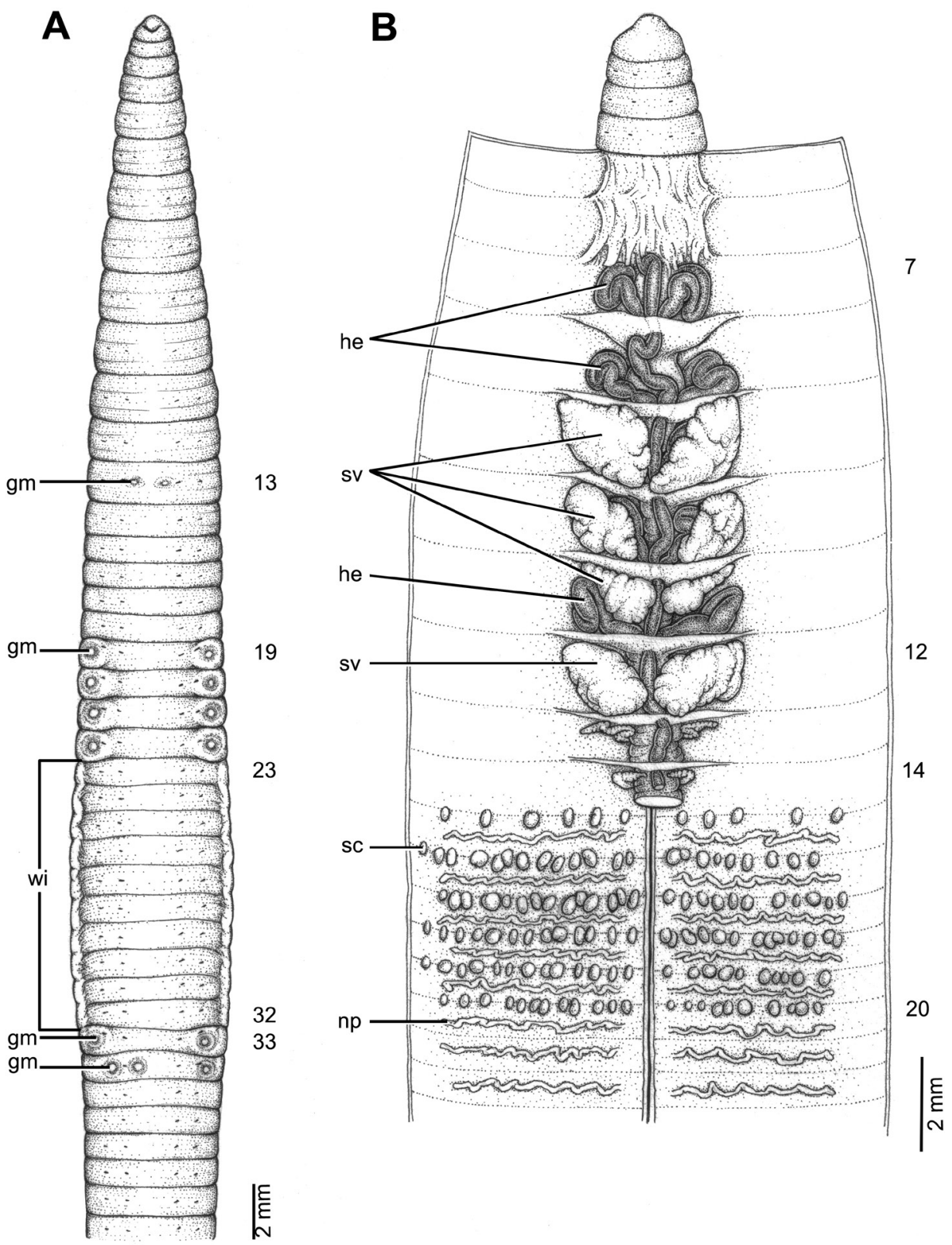

Figure 12. Morphology of holotype (CUMZ 3426) of Glyphidrilus champasakensis sp. n. A external ventral view, annular clitellum in XIX-XLIX B internal dorsal view.

Description of holotype. Dimensions: body length $301 \mathrm{~mm}$, diameter $5.0 \mathrm{~mm}$ in segment VIII, $5.0 \mathrm{~mm}$ before the clitellar wing in segment XXII, $5.0 \mathrm{~mm}$ after wing in segment XXXIII in clitellar region; body cylindrical in anterior part, quadrangular 
in transverse section behind clitellum. 424 segments. Body color pale brown with variations from red to pink at adjacent tissues of wing portion in different individuals of newly collected specimens. Clitellar wing on ventro-lateral part of clitellum in XXIIIXXXII, $10.0 \mathrm{~mm}$ in height, and $0.2 \mathrm{~mm}$ in width on both sides. Prostomium zygolobous. Dorsal pores absent. Clitellum annular in XIX- XLIX. Four pairs of setae per segment from II, setal formula aa:ab:bc:cd:dd = 2.0:1.0:2.0:1.0:3.0 in segment VIII and 2.0:1.0:2.0:1.0:2.5 in postclitellar segments. Female pores, male pores, and spermathecal pores not visible. Genital markings: medium paired or asymmetrical on aa in XIII, XXXIV, laterally paired or asymmetrical on bc in XIX-XXII and XXXIII, XXXIV.

Septa 4/5-8/9 thicker than between other segments others segment, 9/10-11/12 thick and 12/13 to the last segment thin. Gizzard small, globular in VIII. Intestine enlarged from XVI. Hearts, five pairs in VII-XI, first in VII and last in XI. A pair of holonephridia in each segment from segment XIV onwards. Seminal vesicles, four pairs in IX, X, XI, XII. Ovaries, two pairs in XIII and XIV. Testis free in X and XI. Prostate and accessory glands absent. Spermathecae sessile and diverticula absent, small elongated oval or globular between $14 / 15-19 / 20$, about $0.1-0.3 \mathrm{~mm}$ in diameter, five to thirteen on each side per segment.

Variations. Body lengths of adult $(\mathrm{n}=24)$ ranged from $167-301 \mathrm{~mm}(212.75 \pm$ 42.15), with 248-424 segments. Wings in XXIII, XXIV-1/2XXXII, XXXII, XXXIII, clitellum in XIX, XX- XLIX, L, LI, LII. Genital markings: paired or asymmetrical on aa in XII, XIII, XXXIII, XXXIV; paired or asymmetrical on bc in XVII, XVIII, XIXXXII, XXIII, XXIV and XXXII, XXXIII, XXXIV, XXXV.

Distribution. The new species was found only on the banks of the Mekong River at Ban Khonkhen, Champasak, Laos.

Etymology. The new species was named after Champasak, Laos, the type locality.

Remarks. Here we compare Glyphidrilus champasakensis sp. n. to the species with spermathecae and a clitellum beginning from segment XIX. It differs from G. namdonensis sp. n. by G. namdonensis sp. n. having shorter wings in XXIV, XXV-XXVIII, XXX, and spermathecae between 14/15-18/19 (see Table 1).

\section{Discussion}

Semi-aquatic earthworms of the genus Glyphidrilus are widely recorded on the Asian and African continents. Recently, many species have been described from several ecotone areas in Thailand between freshwater and terrestrial habitats of many river basins, where the soil $\mathrm{pH}$ varies from neutral to basic at 7-7.5 (Chanabun et al. 2013).

The seven new species from Thailand and Laos presented in this paper range in size, with respect to the other Glyphidrilus members, from large to small, with $G$. champasakensis sp. n. being the longest and $G$. nanensis sp. n. the smallest. The other five species are almost of the same size, as shown in Table 1 . However, the locations of clitellum, wings, and spermathecae and other characters of the seven species show clear differences from the closely related species. 
Glyphidrilus chiangraiensis sp. n. from Mekong River at Wat Hatkai, Chiangkhong, Chiangrai, north Thailand, was found in many areas of the Mekong River and its tributaries from Thailand and Laos, at elevations from 101-385 meters, and co-existing with G. mekongensis Panha \& Chanabun, 2012, Amynthas mekongianus (Cognetti, 1922), Amynthas sp., and Metaphire sp. The earthworms and cocoons were found in the soil when digging (Fig. 13) and this is probably the cause of the wide distribution, since cocoons travelled from the high to low elevations during the rainy season. Glyphidrilus nanensis sp. n. was found in the rice fields of a tributary of the Nan River, in north Thailand. It was found co-existing with the common terrestrial earthworm Drawida beddardi (Rosa, 1890). Glyphidrilus nanensis sp. n. is probably an isolated endemic occurring in the Nan River valley. This earthworm was found at soil depths of $5-10 \mathrm{~cm}$ in agricultural land. This demonstrates that the species is compatible with at least organic farming and that conservation of the species is not difficult, which is increasingly important in the world. Jouquet et al. (2008b) showed the casts produced by Glyphidrilus sp. can be considered as patches of nutrients in paddy fields. Owa et al. (2003) also observed rice development and greater productivity when earthworm casts were associated to rice plants. Choosai et al. (2010) observed rice development in Northeast Thailand and confirmed the positive effect of earthworms on soil properties, rice yield was higher when presence of casts. This strongly suggests that any agricultural system promoting earthworm development, thereby increasing the number of casts per rice field, could be considered as an useful approach for the sustainable management of paddy fields.

Glyphidrilus satunensis sp. n. was found at a pond, part of a wildlife sanctuary at Satun, south Thailand. It has a similar body size with another species described from nearby in the Malay Peninsula (Chanabun et al. 2013); however, the locations of clitellum, wings, and spermathecae show clear differences between the closely related species.

The four new species described from Laos, G. namphao sp. n., G. sekongensis sp. n., G. namdonensis sp. n., and G. champasakensis sp. n. live in different habitats. Glyphidrilus namphao sp. $\mathrm{n}$. lives in Phao River the river near to the Namphao border between Laos and Vietnam at a higher elevation ( 525 meters) than the other three new species. Glyphidrilus sekongensis sp. n. lives in a stream located within an evergreen and mossy forest at Ban Kiangkong, Lamarm, Sekong; the forest is covers $70 \%$ of the area near the stream. The soils are slightly sandy and black with the accumulation of organic matter. Glyphidrilus namdonensis sp. n. lives in the sandy banks of the Don River, which is one of the branches of Mekong River at Thathek, Khammouan, Laos. Glyphidrilus champasakensis sp. $\mathrm{n}$. is one of the new species along the Mekong River found at a soil depth of 20-40 cm, and has similar characteristics to two other new species (G. sekongensis sp. n. and G. namdonensis sp. n.).

The following key includes only the species most closely related to those described here. The basis for including the species covered in the key is the same as the basis for including previously described species for comparison in the Remarks sections. The remaining species of Glyphidrilus must be identified with other resources. 

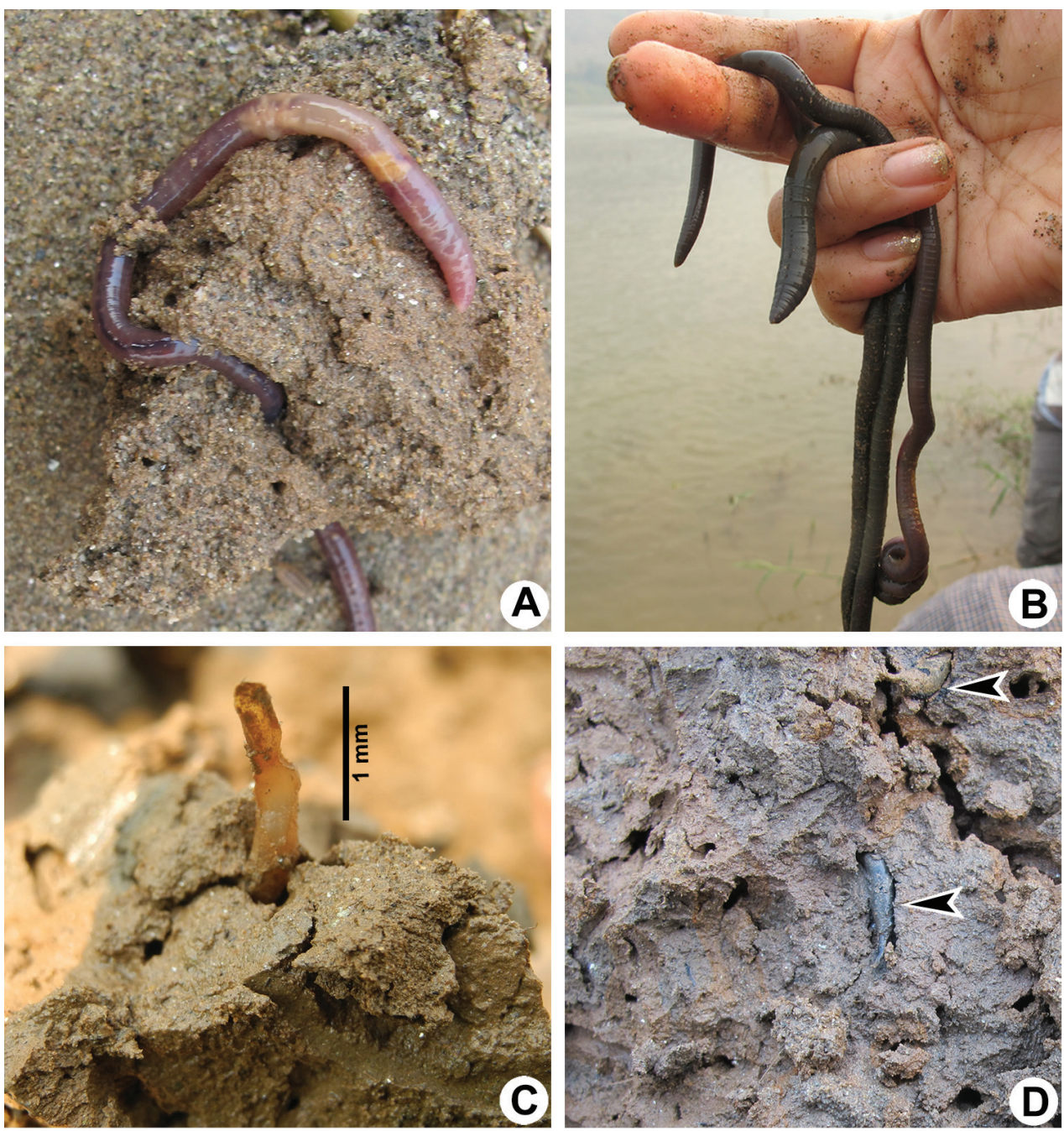

Figure 13. Photographs showing A Metaphire sp. B Amynthas mekongianus (Cognetti, 1922) $\mathbf{C}$ and $\mathbf{D}$ Cocoons of Glyphidrilus in the soil.

Key to species of the newly described species of Glyphidrilus, and those most similar to them

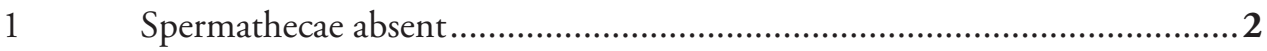

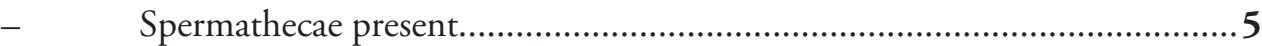

2 Wings in XXII-XXXII, and clitellum in XVIII-XXXVIII ............................

Wings from XXIV or XXV ........................................................... 3 
Clitellum in XII, XIII, XVI-XXXII, XXXIII, wings in XXV, XXVI-XXX, XXXI. G. huailuangensis Chanabun \& Panha, 2013

Clitellum from XIX or XX.

Wings in XXIV-1/2XXXIII, XXXIII, XXXIV, $1 / 2 X X X V$, clitellum in XIXXXXVII, XXXVIII, and intestine beginning from XV ...

G. mekongensis Panha \& Chanabun, 2012 Wings in XXIV, XXV-XXXI, XXXII, clitellum in XIX, XX-XXXV, XXXVI, XXXVII and intestine beginning from XVI

G. vangviengensis Chanabun \& Panha, 2011

Heart in VII-XI.

Heart in VIII-XI

Wings in XVIII-XXIV, clitellum in XVII-XXVI, XXIX, and spermathecae between 13/14-16/17 G. namphao sp. n.

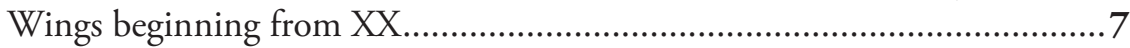

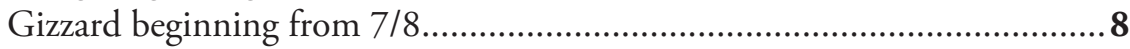

Gizzard beginning in segment VIII ........................................................10 Wings in XXI, XXII-XXVII, XXVIII, XXIX, clitellum in XIV, XVI, XVIIXXXI, XXXII, XXXIII, XXXIV, XXXV, XXXVI and spermathecae between $14 / 15-18 / 19$ G. borealis Chanabun \& Panha, 2013

Wings beginning from XXIV or XXV or XXVI

Wings in XXIV, XXV, XXVI-XXXI, XXXII, clitellum in XII, XIII, XIV, XV, XVI-XL, XLI, XLII and spermathecae between 12/13-18/19

G. vangthongensis Chanabun \& Panha, 2013 Wings in XXIV, XXV, XXVI-XXVII, XXVIII, XXIX, clitellum in XVII, XVIII-XXXIII, XXXIV and spermathecae between 13/14-17/18

G. nanensis sp. n.

Intestine beginning from XV

Intestine beginning from XVI

Spermathecae between $12 / 13$.

Spermathecae between 16/17-22/23, wings in XXIV, XXV-XXXII, XXXIII and clitellum in XX-XLIII, XLIV, XLV .

G. chaophraya Chanabun \& Panha, 2013 Wings in XXIII, XXIV, XXV, XXVI-XXIX, XXX, XXXI, XXXII, clitellum in XVII, XVIII-XXXIII, XXXIV, XXXV, XXXVI, XXXVII, XXXVIII and spermathecae between $12 / 13-18 / 19$. G. chiensis Chanabun \& Panha, 2013 Wings in XXIII, XXIV-XXVIII, XXIX, XXX, XXXI, clitellum in XV, XVI, XVII, XVIII-XXXI, XXXII, XXXIII, XXXIV, XXXV, XXXVI and spermathecae between $12 / 13-17 / 18$. G. quadratus Chanabun \& Panha, 2013 Spermathecae begin from $14 / 15$ Spermathecae between 15/16-20/21, wings in XXIII, XXIV-XXVI, 1/2XXVII, XXVII, 1/2XXVIII, XXVIII and clitellum in XVII, XVIII, XIX, XX, XXI-XXXVI, XXXVII, XXXVIII, XXXIX G. chiangraiensis sp. $\mathrm{n}$. 
14 Wings in XXIV, XXV-XXVIII, XXX, clitellum in XIX, XX-XXXVI, XXXVII, XXXVIII and spermathecae between 14/15-18/19.......G. namdonensis sp. n.

- $\quad$ Wings in XXIII, XXIV-1/2XXXII, XXXII, XXXIII, clitellum in XIX, XX- XLIX, L, LI, LII and spermathecae between 14/15-19/20 .... G. champasakensis sp. n.

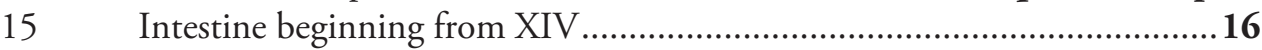

- Intestine beginning from XVI...............................................................

16 Wings in XX, XXI-XXVI, XXVII, clitellum in XI, XII, XIII-XXXIII, XXXIV, XXXV and spermathecae between 13/14-17/18

G. wararamensis Chanabun \& Panha, 2013

- Wings in XXIII, XXIV-XXVIII, XXIX, XXX, clitellum in XVIII-XXX, XXXI, XXXII and spermathecae between 14/15-17/18.

G. kratuensis Chanabun \& Panha, 2013

17 Spermathecae intrasegmental in XVIII-XXI, wings in XXII, XXIII-XXVII, XXVIII and clitellum in XVII, XVIII-XXX

G. trangensis Chanabun \& Panha, 2013

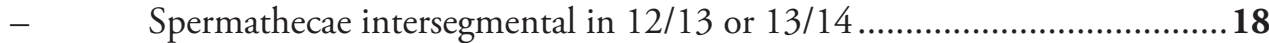

18 Wings in XXIV, XXV, XXVI-XXIX, XXX, XXXI, clitellum in XVII, XVIIIXXXII, XXXIII, XXXIV, XXXV and spermathecae between 13/14-15/16...

G. satunensis sp. $\mathrm{n}$.

- Wings in XXV-XXXI, clitellum in XVI, XVII-XXXVI, XXXVII and spermathecae between $12 / 13-15 / 16$ G. sekongensis sp. $\mathrm{n}$.

\section{Acknowledgements}

This project was partly funded by Chulalongkorn University Graduate School Postdoctoral Project to RC, while most of the financial support was received from The Thailand Research Fund, The TRF Senior Research Scholar RTA 5880002 (2015-2018) to SP. This research was also partially funded by a grant from WCU-058-016-FW (Food and Water Research Cluster, Chulalongkorn University), Higher Education Research Promotion (HERP; Project Code 2559A15562001). We are grateful to Professor Peter K.L. $\mathrm{Ng}$, Dr. Daren Yeo and Dr. Kelvin Tan (ZRC, Singapore), and to Ms. Emma Sherlock (NHMUK, London) and Dr. Andreas Schmidt-Rhaesa (ZMH, Germany) for kindly permitting us to study the type specimens and relevant reference material. Thanks also to Ms. Thita Krutchuen for excellent drawings, and to all members of the Animal Systematics Research Unit, Chulalongkorn University for assistance in collecting material.

\section{References}

Chanabun R, Bantaowong U, Sutcharit C, Tongkerd P, Inkavilay K, James SW, Panha S (2011) A new species of semi-aquatic freshwater earthworm of the genus Glyphidrilus Horst, 1889 from Laos (Oligochaeta: Almidae). Tropical Natural History 11: 213-222. 
Chanabun R, Bantaowong U, Sutcharit C, Tongkerd P, James SW, Panha S (2012a) A new species of semi-aquatic freshwater earthworms of the genus Glyphidrilus Horst, 1889 from the Mekong River (Oligochaeta: Almidae). Raffles Bulletin of Zoology 60(2): 265-277.

Chanabun R, Sutcharit C, Tongkerd P, Shau-Hwai AT, Panha S (2012b) Three new species of semi-aquatic freshwater earthworms of the genus Glyphidrilus Horst, 1889 from Malaysia (Clitellata: Oligochaeta: Almidae). Zootaxa 3458: 120-132.

Chanabun R, Sutcharit C, Tongkerd P, Panha S (2013) The semi-aquatic freshwater earthworms of the genus Glyphidrilus Horst, 1889 from Thailand (Oligochaeta: Almidae) with re-descriptions of several species. ZooKeys 265: 1-76. https://doi.org/10.3897/zookeys.265.3911

Chanabun R, Panha S (2015) Two New Species of Semi-aquatic Earthworms Genus Glyphidrilus Horst, 1889 from Malaysia (Oligochaeta: Almidae). Tropical Natural History 15(2): 179-189.

Choosai C, Jouquet P, Hanboonsong Y, Hartmann C (2010) Effects of earthworms on soil properties and rice production in the rained paddy fields of Northeast Thailand. Applied Soil Ecology 45: 298-303. https://doi.org/10.1016/j.apsoil.2010.05.006

Gates GE (1972) Burmese earthworms. An introduction to the systematics and biology of megadrile oligochaetes with special reference to the Southeast Asia. Transactions of the American Philosophical Society 62: 1-326. https://doi.org/10.2307/1006214

Horst R (1889) Over eene nieuwe soort order de Lumbricinen door Prof. Max Weber uit nedenl. India medegebracht. Tijdschrift der Nederlandsche Dierkundige Vereeniging 2: 77.

Horst R (1893) Earthworms from Malay Archipelago. Zoologische Ergebnisse einer Reise in Niederlandisch Ost Indien 3: 28-83.

Jamieson BGM (1968) A new species of Glyphidrilus (Microchaetidae: Oligochaeta) from East Africa. Journal of Natural History 2:387-395. https://doi.org/10.1080/00222936800770381

Jirapatrasilp P, Prasankok P, Chanabun R, Panha S (2015) Allozyme data reveal genetic diversity and isolation by distance in sympatric Glyphidrilus Horst, 1889 (Oligochaeta: Almidae) of the Lower Mekong River Basin. Biochemical Systematics and Ecology 61: 35-43. https://doi.org/10.1016/j.bse.2015.05.003

Jirapatrasilp P, Prasankok P, Sutcharit C, Chanabun R, Panha S (2016) Two new Cambodian semi-aquatic earthworms in the genus Glyphidrilus Horst, 1889 (Oligochaeta, Almidae), based on morphological and molecular data. Zootaxa 4189(3): 543-558. https://doi. org/10.11646/zootaxa.4189.3.5

Jouquet P, Hartmann C, Choosai C, Hanboonsong Y, Bruner D, Montoroi JP (2008b) Different of earthworms and ants on soil properties of paddy fields in North-East Thailand. Paddy and Water Environment 6: 381-386. https://doi.org/10.1007/s10333-008-0134-x

Michaelsen W (1896) Oligochaeten. Ergebnisse einer zoologishen Forschungsreise in den Molukken und in Borneo, im Auftrage der Senckenbergischen naturforschenden Gesellschaft ausgeführt von Dr. Willy Kükenthal. Abhandlungen Senckenbergischen Naturforschenden Gesellschaft 23: $193-243$.

Michaelsen W (1897) Neue und wenig bekannte afrikanische Terricolen. Mittheilungeb aus dem Naturhistorischen Museum 14: 1-71.

Michaelsen W (1900) Oligochaeta. Das Tierreich 10: 1-575. 
Michaelsen W (1902) Neue Oligochaeten und neue Fundorte alt-bekannter. Jahrbuch der Hamburgischen Wissenschaftlichen Anstalten 19: 1-54.

Michaelsen W (1910) Die Oligochätenfauna der vonderindisch-ceylonischen Region. Abhandulngen aus dem Gebiete der Naturwissenschaften 19: 1-108.

Michaelsen W (1918) Die Lumbriciden, mit besonderer Berücksichtigung der bisher als Familie Glossoscolecidae zusammenfassten Unterfamilien. Zoologische Jahrbücher, Abteilung für Systematik 41: 1-398.

Michaelsen W (1922) Oligochäten aus dem Rijks Museum van Natuurlijke Historie zu Leiden. Capita Zoologica 1: 1-68.

Owa SO, Oyenusi AA, Joda AO, Morafa SOA, Yeye JA (2003) Effect of earthworm casting on growth parameters of rice. African Zoology 38: 229-233.

Rao N (1922) Some new species of earthworms belonging to the genus Glyphidrilus. Annals and Magazine of Natural History 9: 51-68. https://doi.org/10.1080/00222932208632640 Shen HP, Yeo DCJ (2005) Terrestrial earthworms (Oligochaeta) from Singapore. The Raffles Bulletin of Zoology 53: 13-33. 